\title{
Poisson Manifolds, Lie Algebroids, Modular Classes: a Survey
}

Yvette KOSMANN-SCHWARZBACH

Centre de Mathématiques Laurent Schwartz, École Polytechnique, 91128 Palaiseau, France E-mail: yks@math.polytechnique.fr

URL: http://www.math.polytechnique.fr/ kosmann/

Received August 31, 2007, in final form January 02, 2008; Published online January 16, 2008

Original article is available at http://www.emis.de/journals/SIGMA/2008/005/

\begin{abstract}
After a brief summary of the main properties of Poisson manifolds and Lie algebroids in general, we survey recent work on the modular classes of Poisson and twisted Poisson manifolds, of Lie algebroids with a Poisson or twisted Poisson structure, and of Poisson-Nijenhuis manifolds. A review of the spinor approach to the modular class concludes the paper.
\end{abstract}

Key words: Poisson geometry; Poisson cohomology; modular classes; twisted Poisson structures; Lie algebroids; Gerstenhaber algebras; Lie algebroid cohomology; triangular $r$-matrices; quasi-Frobenius algebras; pure spinors

2000 Mathematics Subject Classification: 17B70; 17B56; 17B66; 53C15; 58A10; 15A66; 17B81; 17-02; 53-02; 58-02

Dedicated to the memory of Thomas Branson

\section{Introduction}

The aim of this article is to survey recent work on modular classes in Poisson and Lie algebroid theory, after briefly recalling basic definitions concerning Poisson manifolds, Lie algebroids and bialgebroids, and Gerstenhaber algebras.

Poisson manifolds are smooth manifolds with a Poisson bracket on their ring of functions (see [118] and Section 1.1). Since their introduction by Lichnerowicz in 1977 [88], Poisson geometry and the cohomology of Poisson manifolds have developed into a wide field of research. Lie algebroids are vector bundles with a Lie bracket on their space of sections (see [93, 94, 16] and Section 1.2). Fundamental examples of Lie algebroids include both the tangent bundles of smooth manifolds with the usual Lie bracket of vector fields, and finite-dimensional Lie algebras, which can be considered as Lie algebroids over a point. Lie algebroids were first introduced by Pradines in 1967 [108] as the infinitesimal objects associated with the differentiable groupoids of Ehresmann [32] later called Lie groupoids. They appear most prominently in Poisson geometry, as shown by Coste, Dazord and Weinstein [23], in the theory of group actions and in connection theory $[93,94]$, in foliation theory $[101,36]$, in sigma-models (see, e.g., $[10,81,127,106]$ ), and in many other situations. Lie-Rinehart algebras, known under many different names $[104,74,53]$, are algebraic analogues of Lie algebroids.

The definition of the modular class of a Poisson manifold first appeared in 1985 in the work of Koszul [80], who did not give it a name, and, later, in the work of Weinstein [124]: he demonstrated the relationship of this notion to the group of modular automorphisms of von Neumann algebras, whence the name he proposed, proved many properties of the modular vector fields of Poisson manifolds, and indicated how to generalize the modular class to Lie

\footnotetext{
${ }^{\star}$ This paper is a contribution to the Proceedings of the 2007 Midwest Geometry Conference in honor of Thomas P. Branson. The full collection is available at http://www.emis.de/journals/SIGMA/MGC2007.html
} 
algebroids. The theory was then developed in complex analytic geometry by Brylinski and Zuckerman [13], in algebraic geometry by Polishchuk [107], in the framework of Lie-Rinehart algebras by Huebschmann [54, 55], and in that of Lie algebroids by Evens, Lu and Weinstein [34] who formally defined the modular class of a Lie algebroid, by Ping Xu [125], and by myself [68]. The modular class of a Poisson manifold is the obstruction to the existence of a density invariant under the flows of all Hamiltonian vector fields. In the case of the Lie algebroid associated with a foliation with an orientable normal bundle, the modular class is the obstruction to the existence of an invariant transverse measure ${ }^{1}$. In the case of a Lie algebra considered as a Lie algebroid over a point, the modular class is the trace of the adjoint representation, the infinitesimal modular character. This is another justification for the terminology.

One can consider both the theory of Poisson manifolds and that of Lie algebras with a triangular $r$-matrix, i.e., a skew-symmetric solution of the classical Yang-Baxter equation, as particular cases of the more general notion of Lie algebroid with a Poisson structure. To each of these theories, there corresponds, more generally, a twisted version. Twisted Poisson manifolds were first considered in some quantization problems in string theory $[22,105]$, then they were defined as geometric objects $[62,113]$, and finally viewed as particular cases of Lie algebroids with twisted Poisson structures [111, 72]. Just as a Lie algebra with a triangular $r$-matrix gives rise to a Lie bialgebra in the sense of Drinfeld [29], a Lie algebroid with a Poisson structure gives rise to a Lie bialgebroid in the sense of Mackenzie and Xu [95, 64], while a twisted Poisson structure gives rise to a quasi-Lie-bialgebroid ${ }^{2}$. The problem of defining the modular class of a manifold with a twisted Poisson structure, and more generally that of a Lie algebroid with a twisted Poisson structure, was solved in [72]. (See also a different approach in the unpublished manuscript [7].)

For a Poisson manifold, the modular class coincides up to a factor 2 with the modular class of its cotangent Lie algebroid (Theorem 1 in Section 4.4), and the same is true for a twisted Poisson manifold (Section 7.2), but this simple relation is no longer valid in general for Poisson or twisted Poisson structures on Lie algebroids, in particular on Lie algebras. It was the concept of the modular class of a morphism of Lie algebroids, also called a relative modular class, introduced in [45] and [77], that provided a key for a result valid in this general case (Theorem 2 in Section 5.2, and Section 7.2). Further results were established in [78] concerning the case of the regular Poisson and twisted Poisson structures, whose image remains of constant rank, with applications to Lie algebra theory (see Section 6).

In Section 1, we recall the main definitions concerning Poisson manifolds and Lie algebroids, and we show how the two notions are connected (Section 1.4). The modular class of a Poisson manifold is studied in Section 2, that of a Lie algebroid with a Poisson structure in Section 3, with further results in Section 5.2. Section 4 deals with the case of Lie algebroids in general, and Theorem 1 relates the modular class of a Poisson manifold to that of its cotangent Lie algebroid. In Section 5, we introduce the notion of modular class of a Lie algebroid morphism which enables us to state Theorem 2, relating the modular class of a Lie algebroid with a Poisson structure to that of the Lie algebroid morphism defined by the Poisson bivector. In Section 6 , we summarize results pertaining to the case of regular Poisson structures, where simplifications occur in the computation of the modular class (Theorem 3), and we describe the case of quasiFrobenius and Frobenius Lie algebras for which the modular class is computed by a simple

\footnotetext{
${ }^{1}$ More generally, to each Lie algebroid is associated a singular foliation whose leaves are integral manifolds of the image of its anchor - a generalized distribution on the base manifold -, and the vanishing of the modular class generalizes the existence of an invariant transverse measure. Lie algebroids are the setting in which singular foliations can be studied: the holonomy and the linear holonomy of Lie algebroids introduced by Fernandes [36, 37] defined in terms of $A$-paths, paths whose image under the anchor map is the tangent to their projection on the base manifold, generalize the holonomy and linear holonomy of foliations.

${ }^{2}$ The various generalizations of Lie bialgebras were first considered and studied in [63], following the introduction of the Lie-quasi-bialgebras as the semi-classical limits of quasi-Hopf algebras by Drinfeld [30], while the algebroid generalization is due to Roytenberg [111].
} 
formula (Theorem 4). In Section 7, we have collected results concerning the twisted case: the definition of a twisted Poisson structure and its modular class, the statement of Theorem 5 in Section 7.2 which gives an explicit representative of the modular class, the study of the non-degenerate case with the linearization of the twisted Poisson condition into the twisted symplectic condition in Section 7.4, and an example of a twisted triangular $r$-matrix given in Section 7.5. Section 8 deals with the modular classes of Poisson-Nijenhuis manifolds and their relation with the bi-Hamiltonian hierarchy of the Poisson-Nijenhuis structure (Theorem 6). In Section 9, which is the last, we explain another characterization of the modular class that has recently appeared, the spinor approach to Dirac structures in Courant algebroids. Theorem 7 in Section 9.1 can be viewed as an alternative definition of the modular class in the twisted case that confirms that the original definition in [72] is the right one. In the Appendix, we indicate additional references to recent publications and work in progress.

Whereas the computation of the Poisson cohomology ring of a Poisson manifold or the Lie algebroid cohomology ring of a Lie algebroid is a very difficult task, there are explicit formulas for computing the modular class which is the subject of this survey. The modular class is but the first in the sequence of higher characteristic classes of Lie algebroids studied by Kubarski (see [82]), by Fernandes [35, 36, 37] and by Crainic [24], and in the framework of generalized gauge theories by Lyakhovich and Sharapov [92]. The generalization of the properties of the modular class to the higher order classes is an open problem.

Most, but not all, of the results in this survey are to be found in several papers written with Camille Laurent-Gengoux, Franco Magri, Alan Weinstein and Milen Yakimov. I have tried to give due credit to them and to the other authors whose work I have consulted. But the literature has become so vast, that I must be forgiven for not having mentioned all the relevant papers.

\section{Conventions}

All manifolds and bundles are assumed to be smooth, and spaces of sections, denoted $\Gamma(\cdot)$, are spaces of smooth sections. Forms (resp., multivectors) on a vector bundle $E$ are sections of $\wedge \bullet E^{*}$ (resp., sections of $\wedge^{\bullet} E$ ). We use the notations $\iota$ and $\epsilon$ for the operators of interior and exterior multiplication on forms and multivectors, with the convention $\iota_{X \wedge Y}=\iota_{X} \circ \iota_{Y}$. A derivation of a graded algebra means a graded derivation. A biderivation of a bracket means a graded derivation in each argument.

\section{$1 \quad$ Lie algebroids and Poisson geometry}

\subsection{Poisson manifolds}

Let us recall the definition of a Poisson manifold ${ }^{3}$. When $M$ is a smooth manifold and $\pi$ is a bivector field, i.e., a contravariant, skew-symmetric, 2-tensor field, $\left.\pi \in \Gamma\left(\wedge^{2} T M\right)\right)$, define $\{f, g\}$ as $\pi(\mathrm{d} f, \mathrm{~d} g)$, for $f$ and $g \in C^{\infty}(M)$. The pair $(M, \pi)$ is called a Poisson manifold if $\{$,$\} is an \mathbb{R}$-Lie bracket. When $(M, \pi)$ is a Poisson manifold, $\pi$ is called a Poisson bivector or a Poisson structure on $M$ and $\{$,$\} is called the Poisson bracket. Examples are (i) symplectic$ structures: if $\omega$ is a non-degenerate closed 2-form on $M$, then its inverse is a Poisson bivector, and (ii) the duals of Lie algebras: if $\mathfrak{g}$ is a Lie algebra, then the Poisson bracket on $\mathfrak{g}^{*}$ is defined by $\{x, y\}(\xi)=\langle\xi,[x, y]\rangle$, where $x$ and $y \in \mathfrak{g}$ are considered as linear forms on $\mathfrak{g}^{*}$, and $\xi \in \mathfrak{g}^{*}$. This linear Poisson structure on $\mathfrak{g}^{*}$ is called the Kirillov-Kostant-Souriau Poisson structure.

\footnotetext{
${ }^{3}$ Lie's function groups, further studied by Carathéodory, were precursors of Poisson manifolds. Poisson manifolds, which occur already in [38], were defined by Lichnerowicz in [88]. Closely related notions also appeared in the work of Berezin, Hermann, Tulczyjew and Kirillov, while Poisson structures on infinite-dimensional manifolds were introduced under the name Hamiltonian structures by several authors working on integrable systems. For an exposition of Poisson geometry, see [87, 118, 94].
} 
We shall call bivector fields simply bivectors, and, more generally, we shall call the sections of $\wedge^{\bullet}(T M)$ multivectors. The space of multivectors is an associative, graded commutative algebra under the exterior multiplication and it is endowed with a graded Lie algebra structure (for a shifted grading) called the Schouten-Nijenhuis bracket or the Schouten bracket, making it a Gerstenhaber algebra (see Section 1.3 below). We shall denote the $(k+\ell-1)$-vector which is the Schouten-Nijenhuis bracket of a $k$-vector $U$ and an $\ell$-vector $V$ on $M$ by $[U, V]_{M}$. The Schouten-Nijenhuis bracket of multivectors is the extension of the Lie bracket of vector fields as a biderivation, satisfying $[X, f]_{M}=X \cdot f$, for $X \in \Gamma(T M), f \in C^{\infty}(M)$. The following fundamental fact is easy to prove.

Proposition 1. A bivector $\pi$ is a Poisson structure on $M$ if and only if $[\pi, \pi]_{M}=0$.

When $\pi$ is a bivector, we define $\pi^{\sharp}: T^{*} M \rightarrow T M$ by

$$
\pi^{\sharp}(\alpha)=\iota_{\alpha} \pi,
$$

where $\alpha \in T^{*} M$, and $\iota_{\alpha}$ denotes the interior product of multivectors by the 1-form $\alpha$.

The Hamiltonian vector field associated to $f \in C^{\infty}(M)$ is

$$
H_{f}^{\pi}=\pi^{\sharp}(\mathrm{d} f) .
$$

Associated to any Poisson structure $\pi$ on $M$, there is a differential, i.e., an operator of degree 1 and square zero, on $\Gamma\left(\wedge^{\bullet} T M\right)$,

$$
\mathrm{d}_{\pi}=[\pi, \cdot]_{M},
$$

called the Lichnerowicz-Poisson differential or the Poisson cohomology operator. The cohomology of the complex $\left(\Gamma\left(\wedge^{\bullet} T M\right), \mathrm{d}_{\pi}\right)$ is called the Poisson cohomology of $(M, \pi)$. In particular, $H_{f}^{\pi}=-[\pi, f]_{M}=-\mathrm{d}_{\pi} f$.

We also define the Poisson homology operator on $\Gamma\left(\wedge^{\bullet} T^{*} M\right)$ (also called the Koszul-Brylinski homology operator) by

$$
\partial_{\pi}=\left[\mathrm{d}, \iota_{\pi}\right],
$$

where the bracket is the graded commutator of operators, so that $\partial_{\pi}=\mathrm{d} \circ \iota_{\pi}-\iota_{\pi} \circ \mathrm{d}$. It is an operator of degree -1 and square zero. The homology of the complex $\left(\Gamma\left(\wedge^{\bullet} T^{*} M\right), \partial_{\pi}\right)$ is called the Poisson homology of $(M, \pi)$.

\subsection{Lie algebroids}

We now turn to the definition of Lie algebroids ${ }^{4}$, and in Section 1.4 we shall show how Lie algebroids appear in Poisson geometry.

A Lie algebroid structure on a real vector bundle $A \rightarrow M$ is defined by a vector bundle map, $a_{A}: A \rightarrow T M$, called the anchor of $A$, and an $\mathbb{R}$-Lie algebra bracket on $\Gamma A,[,]_{A}$, satisfying the Leibniz rule,

$$
[X, f Y]_{A}=f[X, Y]_{A}+\left(a_{A}(X) \cdot f\right) Y,
$$

for all $X, Y \in \Gamma A, f \in C^{\infty}(M)$. We shall denote such a Lie algebroid by $\left(A, a_{A},[,]_{A}\right)$ or simply by $A$, when there is no risk of confusion. It follows from these axioms that the map $a_{A}$ induces a morphism of Lie algebras from $\Gamma A$ to $\Gamma(T M)$, which we denote by the same symbol, i.e.,

$$
a_{A}\left([X, Y]_{A}\right)=\left[a_{A}(X), a_{A}(Y)\right]_{M},
$$

for all $X, Y \in \Gamma A$. Fundamental examples of Lie algebroids are the following.

\footnotetext{
${ }^{4}$ For an exposition of Lie algebroid theory, see [93, 94] and [16].
} 
Example 1. Any tangent bundle $A=T M$ of a manifold, $M$, with $a_{A}=\operatorname{Id}_{T M}$ and the usual Lie bracket of vector fields, is a Lie algebroid.

Example 2. Any Lie algebra, $A=\mathfrak{g}$, considered as a vector bundle over the singleton $M=\{\mathrm{pt}\}$, with trivial anchor $a_{A}=0$, is a Lie algebroid.

Example 3. Lie algebra bundles (see [93, 94]) are locally trivial Lie algebroids with vanishing anchor. The Lie bracket is defined pointwise and varies smoothly with the base point. The integration of such bundles of Lie algebras into bundles of Lie groups was studied by Douady and Lazard [28].

Proposition 2. Associated to a given Lie algebroid, $\left(A, a_{A},[,]_{A}\right)$, there is a differential, $\mathrm{d}_{A}$, on $\Gamma\left(\wedge^{\bullet} A^{*}\right)$, defined by

$$
\begin{aligned}
\left(\mathrm{d}_{A} \alpha\right)\left(X_{0}, \ldots, X_{k}\right)= & \sum_{i=0}^{k}(-1)^{i} a_{A}\left(X_{i}\right) \cdot \alpha\left(X_{0}, \ldots, \widehat{X}_{i}, \ldots, X_{k}\right) \\
& +\sum_{0 \leq i<j \leq k}(-1)^{i+j} \alpha\left(\left[X_{i}, X_{j}\right]_{A}, X_{0}, \ldots, \widehat{X}_{i}, \ldots, \widehat{X}_{j}, \ldots, X_{k}\right),
\end{aligned}
$$

for $\alpha \in \Gamma\left(\wedge^{k} A^{*}\right), X_{0}, \ldots, X_{k} \in \Gamma A$.

The coboundary operator $\mathrm{d}_{A}$ is called the Lie algebroid differential of $A$. The cohomology of the complex $\left(\Gamma\left(\wedge^{\bullet} A^{*}\right), \mathrm{d}_{A}\right)$, called the Lie algebroid cohomology of $A$, unifies de Rham and Chevalley-Eilenberg cohomologies [104] (see also [53]): for a tangent bundle, $A=T M$, with $a_{A}=\operatorname{Id}_{T M}, \mathrm{~d}_{A}=\mathrm{d}_{M}$ is the de Rham differential, while for a Lie algebra, $A=\mathfrak{g}$, considered as a Lie algebroid over the singleton $M=\{\mathrm{pt}\}, \mathrm{d}_{A}=\mathrm{d}_{\mathfrak{g}}$ is the Chevalley-Eilenberg differential.

We call sections of $\wedge^{\bullet} A$ (resp., $\wedge^{\bullet}\left(A^{*}\right)$ ) multivectors (resp., forms) on $A$.

\subsection{Gerstenhaber algebras and Batalin-Vilkovisky algebras}

Gerstenhaber showed that there is a graded Lie bracket, since called a Gerstenhaber bracket, on the Hochschild cohomology of an associative algebra [41]. We recall the definition of a Gerstenhaber algebra.

A Gerstenhaber algebra is an associative, graded commutative algebra over a field $K$ of characteristic zero, $\left(\mathcal{A}=\oplus_{k \in \mathbb{Z}} \mathcal{A}^{k}, \wedge\right)$, with a graded $K$-Lie algebra structure, [ , ], on $\left(\oplus_{k \in \mathbb{Z}} \mathcal{A}^{k+1}, \wedge\right)$ such that, for $u \in \mathcal{A}^{k},[u, \cdot]$ is a derivation of degree $k-1$ of $\left(\mathcal{A}=\oplus_{k \in \mathbb{Z}} \mathcal{A}^{k}, \wedge\right)$,

$$
[u, v \wedge w]=[u, v] \wedge w+(-1)^{(k-1) \ell} v \wedge[u, w],
$$

for $u \in \mathcal{A}^{k}, v \in \mathcal{A}^{\ell}, w \in \mathcal{A}$. The bracket of a Gerstenhaber algebra is also called an odd Poisson bracket $[117,122]$ or a Schouten bracket [74].

A linear map, $\partial: \mathcal{A} \rightarrow \mathcal{A}$, of degree -1 , is called a generator of the Gerstenhaber bracket if

$$
[u, v]_{\mathcal{A}}=(-1)^{k}\left(\partial(u \wedge v)-\partial u \wedge v-(-1)^{k} u \wedge \partial v\right),
$$

for all $u \in \mathcal{A}^{k}, v \in \mathcal{A}$. If $\partial^{2}=0$, then $(\mathcal{A}, \partial)$ is called a Batalin-Vilkovisky algebra (in short, $\mathrm{BV}$-algebra).

For a Lie algebroid, $A$, there is a Gerstenhaber bracket on $\Gamma\left(\wedge^{\bullet} A\right)$, which generalizes the Schouten-Nijenhuis bracket of multivectors on a manifold:

Proposition 3. When $A$ is a Lie algebroid, $\left(\Gamma(\wedge \bullet A), \wedge,[,]_{A}\right)$, where $[,]_{A}$ is the extension of the Lie bracket on $\Gamma A$ as a biderivation satisfying $[X, f]_{A}=\left\langle X, \mathrm{~d}_{A} f\right\rangle$, for $X \in \Gamma A$ and $f \in C^{\infty}(M)$, is a Gerstenhaber algebra. 
When $A=T M$, for $M$ a manifold, $\Gamma\left(\wedge^{\bullet} A\right)$ is the exterior algebra of multivectors on $M$ and the Gerstenhaber bracket on this algebra is the Schouten-Nijenhuis bracket, which we have introduced in Section 1.1 and denoted there by $[,]_{M}$. When $A=\mathfrak{g}$, a Lie algebra, we recover the algebraic Schouten bracket on $\wedge^{\bullet} \mathfrak{g}$, denoted [, ] , which can be defined as the bracket induced from the Schouten-Nijenhuis bracket of left-invariant multivectors on a Lie group with Lie algebra $\mathfrak{g}$.

In both cases, the Gerstenhaber bracket is a Batalin-Vilkovisky bracket: for a manifold, $M$, transposing the de Rham differential by means of the isomorphism from forms to multivectors defined by a top degree density yields a generator of square zero of the Schouten-Nijenhuis bracket, while the Lie algebra homology operator yields a generator of square zero of the algebraic Schouten bracket of a Lie algebra.

The problem of identifying which Gerstenhaber algebras are Batalin-Vilkovisky algebras is the subject of much recent work. See [98] and references therein.

\subsection{The cotangent bundle of a Poisson manifold is a Lie algebroid}

Let $M$ be a manifold equipped with a bivector $\pi \in \Gamma\left(\wedge^{2} T M\right)$. Define

$$
[\alpha, \beta]_{\pi}=\mathcal{L}_{\pi^{\sharp} \alpha} \beta-\mathcal{L}_{\pi^{\sharp} \beta} \alpha-\mathrm{d}(\pi(\alpha, \beta)),
$$

for $\alpha, \beta \in \Gamma\left(T^{*} M\right)$, where $\mathcal{L}$ denotes the Lie derivation. It is easy to show that this skewsymmetric bracket ${ }^{5}$ satisfies the Jacobi identity if and only if $[\pi, \pi]_{M}=0$. Bracket (1.2) satisfies

$$
[\mathrm{d} f, \mathrm{~d} g]_{\pi}=\mathrm{d}\{f, g\},
$$

for all $f, g \in C^{\infty}(M)$, and it satisfies the Leibniz rule

$$
[\alpha, f \beta]_{\pi}=f[\alpha, \beta]_{\pi}+\left(\pi^{\sharp} \alpha \cdot f\right) \beta,
$$

for all $\alpha, \beta \in \Gamma\left(T^{*} M\right)$ and $f \in C^{\infty}(M)$. It was remarked by Huebschmann [53] that these properties determine the above bracket.

The extension to forms of all degrees on a Poisson manifold of bracket (1.2) is denoted by the same symbol. It is the Koszul bracket [80], which satisfies the relation

$$
[\alpha, \beta]_{\pi}=(-1)^{k}\left(\partial_{\pi}(\alpha \wedge \beta)-\partial_{\pi} \alpha \wedge \beta-(-1)^{k} \alpha \wedge \partial_{\pi} \beta\right),
$$

for all $\alpha \in \Gamma\left(\wedge^{k} T^{*} M\right), \beta \in \Gamma\left(\wedge^{\bullet} T^{*} M\right)$, where $\partial_{\pi}$ is the Poisson homology operator $\left[\mathrm{d}, \iota_{\pi}\right]$. In particular, $[\alpha, f]_{\pi}=\left(\pi^{\sharp} \alpha\right) \cdot f$, for $\alpha \in \Gamma\left(T^{*} M\right)$ and $f \in C^{\infty}(M)$. The following proposition follows from $[80,9,74]$.

Proposition 4. If $(M, \pi)$ is a Poisson manifold, $\left(T^{*} M, \pi^{\sharp},[,]_{\pi}\right)$ is a Lie algebroid. The associated Lie algebroid differential is the Lichnerowicz-Poisson differential on the multivectors on $M, \mathrm{~d}_{\pi}=[\pi, \cdot]_{M}$. The associated Gerstenhaber algebra is the algebra of forms on $M$ with the Koszul bracket, which is a Batalin-Vilkovisky algebra with generator the Poisson homology operator $\partial_{\pi}=\left[\mathrm{d}, \iota_{\pi}\right]$.

In fact, more can be said. There is a compatibility condition between the Lie algebroid structures of $T M$ and $T^{*} M$ making the pair $\left(T M, T^{*} M\right)$ a Lie bialgebroid, a notion which we now define.

\footnotetext{
${ }^{5}$ Formula (1.2) appeared as the bracket of 1-forms on a symplectic manifold in the first edition of the book on mechanics by Abraham and Marsden [2] in 1967, before anyone spoke about Poisson manifolds, and again in [3]; formula (1.3) below is also stated there. Formula (1.2) was re-discovered as the bracket of 1-forms on a Poisson manifold by several authors, Fuchssteiner [39], Gelfand and Dorfman [40], Magri and Morosi [96] and Karasëv [61]. In 1987, Weinstein made the connection between this formula and the theory of Lie groupoids and Lie algebroids (see [23]): the manifold of units $\Gamma_{0}$ of a symplectic groupoid $\Gamma$ is a Poisson manifold, and the Lie algebroid of such a Lie groupoid is canonically isomorphic to the cotangent bundle of $\Gamma_{0}$, so there is a Lie algebroid bracket on the space of sections of the cotangent bundle of $\Gamma_{0}$. A calculation proves that it is given by (1.2), the formula of Abraham and Marsden.
} 


\subsection{Lie bialgebroids}

A Lie bialgebroid $[95,64]$ is a pair of Lie algebroids in duality $\left(A, A^{*}\right)$ such that $\mathrm{d}_{A}$ is a derivation of $[,]_{A^{*}}$. This condition is satisfied if and only if $\mathrm{d}_{A^{*}}$ is a derivation of $[,]_{A}$. Therefore the notion of Lie bialgebroid is self-dual: $\left(A, A^{*}\right)$ is a Lie bialgebroid if and only if $\left(A^{*}, A\right)$ is a Lie bialgebroid.

When $\pi$ is a Poisson bivector on $M$ and $T^{*} M$ is equipped with the Lie algebroid structure defined above, the pair $\left(T M, T^{*} M\right)$ is a Lie bialgebroid: in fact $\mathrm{d}_{T^{*} M}=\mathrm{d}_{\pi}=[\pi, \cdot]_{M}$ is clearly a derivation of the Gerstenhaber bracket, $[,]_{M}$.

When $M$ is a point, the notion of Lie bialgebroid reduces to that of a Lie bialgebra [29] (see also $[86,63])$.

\subsection{Lie algebroids with a Poisson structure}

Lie algebroids with a Poisson structure generalize Poisson manifolds.

Let $\left(A, a_{A},[,]_{A}\right)$ be a Lie algebroid, and assume that $\pi \in \Gamma\left(\wedge^{2} A\right)$ satisfies $[\pi, \pi]_{A}=0$. Then $(A, \pi)$ is called a Lie algebroid with a Poisson structure.

Let us define

$$
[\alpha, \beta]_{\pi}=\mathcal{L}_{\pi^{\sharp} \alpha}^{A} \beta-\mathcal{L}_{\pi^{\sharp} \beta}^{A} \alpha-\mathrm{d}_{A}(\pi(\alpha, \beta)),
$$

for $\alpha, \beta \in \Gamma\left(A^{*}\right)$, where $\mathcal{L}^{A}$ denotes the Lie derivation on sections of $\wedge^{\bullet}\left(A^{*}\right)$ defined by $\mathcal{L}_{X}^{A}=$ $\left[\mathrm{d}_{A}, \iota_{X}\right]$, for $X \in \Gamma A$, and $\pi^{\sharp}: A^{*} \rightarrow A$ is defined by $\pi^{\sharp} \alpha=\iota_{\alpha} \pi$, and set $a_{A^{*}}=a_{A} \circ \pi^{\sharp}$. Then $\left(A^{*}, a_{A^{*}},[,]_{\pi}\right)$ is a Lie algebroid. The associated differential is $\mathrm{d}_{\pi}=[\pi, \cdot]_{A}$, and the Gestenhaber bracket on the sections of $\wedge^{\bullet}\left(A^{*}\right)$, also denoted by $[,]_{\pi}$, satisfies relation (1.5), where $\partial_{\pi}=\left[\mathrm{d}_{A}, \iota_{\pi}\right]$. It is therefore clear that, when $A$ is a Lie algebroid with a Poisson structure, the pair $\left(A, A^{*}\right)$ is a Lie bialgebroid.

The Poisson cohomology $H^{\bullet}\left(A, \mathrm{~d}_{\pi}\right)$ of a Lie algebroid with a Poisson structure $(A, \pi)$ is the cohomology of the complex $\left(\Gamma\left(\wedge^{\bullet} A\right), \mathrm{d}_{\pi}\right)$ which is the cohomology of the Lie algebroid $A^{*}$ with anchor $a_{A} \circ \pi^{\sharp}$ and Lie bracket $[,]_{\pi}$, and which generalizes the Poisson cohomology of Lichnerowicz for Poisson manifolds [88]. The Poisson homology $H_{\bullet}\left(A, \partial_{\pi}\right)$ of $(A, \pi)$ is that of the complex $\left(\Gamma\left(\wedge^{\bullet} A^{*}\right), \partial_{\pi}\right)$ which has been studied by Huebschmann [55], and which generalizes the Poisson homology of Poisson manifolds [80, 12].

If $A=\mathfrak{g}$, a Lie algebra, and $\pi=r \in \wedge^{2} \mathfrak{g}$, the Poisson condition is

$$
[r, r]_{\mathfrak{g}}=0 .
$$

This equation is the classical Yang-Baxter equation (CYBE) which can also be written in tensor notation ([29] and see [19, 67]),

$$
\left[r_{12}, r_{13}\right]+\left[r_{12}, r_{23}\right]+\left[r_{13}, r_{23}\right]=0 \text {. }
$$

If $r \in \wedge^{2} \mathfrak{g}$ satisfies the classical Yang-Baxter equation, it is called a triangular $r$-matrix. As a particular case of the study of Lie algebroids with a Poisson structure, we recover the fact that a triangular $r$-matrix on $\mathfrak{g}$ defines a Lie algebra structure on $\mathfrak{g}^{*}$, by

$$
[\alpha, \beta]_{r}=\operatorname{ad}_{r^{\sharp} \alpha}^{*} \beta-\operatorname{ad}_{r^{\sharp} \beta}^{*} \alpha,
$$

in fact a Lie bialgebra structure on $\left(\mathfrak{g}, \mathfrak{g}^{*}\right)$, called a triangular Lie bialgebra.

In analogy with the case of Lie bialgebras defined by a skew-symmetric solution of the classical Yang-Baxter equation, a Lie bialgebroid defined by a Poisson bivector is also called a triangular Lie bialgebroid [90]. 


\subsection{The big bracket}

This section is a very brief summary of parts of several articles [117, 111, 115, 69]. The term "big bracket" was coined in [63].

Let $A$ be a vector bundle over $M$. The big bracket is the canonical Poisson bracket on the cotangent bundle of the supermanifold $\Pi A$, where $\Pi$ denotes the change of parity [117, 122], generalizing the bracket of $[79,86,63]$. We shall denote it by $\{$,$\} .$

Let $\mu$ denote a Lie algebroid structure of $A$ viewed as a function on $T^{*}(\Pi A)$. It is a cubic element of bidegree $(1,2)$, which is homological, i.e., $\{\mu, \mu\}=0$. Then

$$
[X, Y]_{A}=\{\{X, \mu\}, Y\}
$$

for all $X, Y \in \Gamma\left(\wedge^{\bullet} A\right)$. Thus the Lie algebroid bracket $[,]_{A}$ is a derived bracket of the big bracket in the sense of $[66,69]$.

A Lie bialgebroid is defined by a homological function with terms of bidegrees $(1,2)$ and $(2,1)$.

A Lie-quasi-bialgebroid (resp., quasi-Lie-bialgebroid) is defined by a homological cubic element with no term of bidgree $(3,0)$ (resp., no term of bidegree $(0,3)$ ). Whereas for a Poisson manifold $M$, the pair $\left(T M, T^{*} M\right)$ is a bialgebroid, it is a quasi-Lie-bialgebroid when $M$ is a twisted Poisson manifold (see Section 7.1).

A proto-bialgebroid is defined by a homological cubic element in the algebra of functions on $T^{*}(\Pi A)[111]$ (for the case of Lie algebras, see [63]).

The bracket (1.6) on $\Gamma\left(A^{*}\right)$ defined by a Poisson bivector $\pi$ is $\{\pi, \mu\}$, and the Gerstenhaber bracket $[,]_{\pi}$ on $\Gamma\left(\wedge A^{*}\right)$ satisfies

$$
[\alpha, \beta]_{\pi}=\{\{\alpha, \gamma\}, \beta\},
$$

for all $\alpha, \beta \in \Gamma\left(\wedge^{\bullet} A^{*}\right)$, where $\gamma=\{\pi, \mu\}$. Thus the Gerstenhaber bracket $[,]_{\pi}$ also is a derived bracket of the big bracket.

\section{The modular class of a Poisson manifold}

The modular class of a Poisson manifold is a class in the first Poisson cohomology space of the manifold, i.e., the equivalence class modulo Hamiltonian vector fields of a vector field which is an infinitesimal Poisson automorphism, called a modular vector field. On an orientable manifold, there exists a volume form invariant under all Hamiltonian vector fields if and only if there exists a modular vector field which vanishes.

\subsection{Modular vector fields and modular class}

We consider a Poisson manifold $(M, \pi)$. Recall that we denoted the Hamiltonian vector field with Hamiltonian $f$ by $H_{f}^{\pi}$, that the Poisson cohomology operator is $\mathrm{d}_{\pi}=[\pi, \cdot]_{M}$ and that the Poisson homology operator is $\partial_{\pi}=\left[\mathrm{d}, i_{\pi}\right]$.

Assuming that $M$ is orientable, we choose a volume form, $\lambda$, on $M$. The divergence, $\operatorname{div}_{\lambda} Y$, of a vector field $Y$ with respect to $\lambda$ is defined by $\mathcal{L}_{Y} \lambda=\left(\operatorname{div}_{\lambda} Y\right) \lambda$. Let us consider the linear map,

$$
X_{\lambda}: f \mapsto \operatorname{div}_{\lambda}\left(H_{f}^{\pi}\right) .
$$

Then

Proposition 5. $-X_{\lambda}$ is a derivation of $C^{\infty}(M)$, i.e., a vector field on $M$,

- $X_{\lambda}$ is a 1-cocycle in the Poisson cohomology of $(M, \pi)$,

- the Poisson cohomology class of $X_{\lambda}$ is independent of the choice of volume form, $\lambda$. 
Definition 1. The vector field $X_{\lambda}$ is called a modular vector field of $(M, \pi)$. The $\mathrm{d}_{\pi}$-cohomology class of $X_{\lambda}$ is called the modular class of the Poisson manifold $(M, \pi)$.

We shall denote the modular class of $(M, \pi)$ by $\theta(\pi)$. The property $\mathrm{d}_{\pi} X_{\lambda}=0$ means that $\mathcal{L}_{X_{\lambda}} \pi=0$, i.e., that the modular vector fields are infinitesimal automorphisms of the Poisson structure.

When $M$ is not orientable, densities are used instead of volume forms in order to define the modular class.

Example 4. If $(M, \pi)$ is symplectic, then $\theta(\pi)=0$. In fact, the Liouville volume form is invariant under all Hamiltonian vector fields.

If $M=\mathfrak{g}^{*}$, where $\mathfrak{g}$ is a Lie algebra, is equipped with the linear Poisson structure (KirillovKostant-Souriau Poisson structure), the modular vector field associated with the standard Lebesgue measure, $\lambda$, on the vector space, $\mathfrak{g}^{*}$, is the constant vector field on $\mathfrak{g}^{*}$, i.e., linear form on $\mathfrak{g}, X_{\lambda}=\operatorname{Tr}(\mathrm{ad})$. Thus the modular vector field of the Poisson manifold $\mathfrak{g}^{*}$ is the infinitesimal modular character of $\mathfrak{g}$.

Example 5. Consider the 2-dimensional non-Abelian Lie algebra $\mathfrak{g}$, with basis $\left(e_{1}, e_{2}\right)$ and commutation relation, $\left[e_{1}, e_{2}\right]=e_{2}$. The trace of the adjoint representation of $\mathfrak{g}$ is the 1 -form on $\mathfrak{g}, e_{1}^{*}$. In the dual vector space $\mathfrak{g}^{*} \simeq \mathbb{R}^{2}$, with dual basis $\left(u_{1}, u_{2}\right)$, where $u_{1}=e_{1}^{*}, u_{2}=e_{2}^{*}$, and coordinates $\left(\xi_{1}, \xi_{2}\right)$, the linear Poisson structure is defined by the bivector $\pi$ such that $\pi_{\left(\xi_{1}, \xi_{2}\right)}=\xi_{2} u_{1} \wedge u_{2}$. The constant vector field $u_{1}$ is the modular vector field of $\left(\mathfrak{g}^{*}, \pi\right)$ with respect to the measure $\mathrm{d} \xi_{1} \wedge \mathrm{d} \xi_{2}$. Since $u_{1}$ is not globally Hamiltonian, the modular class of $\left(\mathbb{R}^{2}, \pi\right)$ is non-vanishing.

We have thus given an example of a Poisson structure on $\mathbb{R}^{2}$ whose modular class is nonvanishing. See $[109,110]$ for examples of Poisson structures with non-vanishing modular class on surfaces.

\subsection{Properties of modular vector fields}

We state without proof the main properties of the modular vector fields of Poisson manifolds (see $[124,125,68])$. Each of the properties listed below can be adopted as a definition of the modular vector field, $X_{\lambda}$.

- For all $\alpha \in \Gamma\left(T^{*} M\right)$,

$$
\left\langle\alpha, X_{\lambda}\right\rangle \lambda=\mathcal{L}_{\pi^{\sharp} \alpha} \lambda-\left(\iota_{\pi} \mathrm{d} \alpha\right) \lambda,
$$

a relation which reduces to $(2.1), X_{\lambda}(f)=\operatorname{div}_{\lambda}\left(H_{f}^{\pi}\right)$, when $\alpha=\mathrm{d} f$.

- Let $n$ be the dimension of $M$. Given a volume form, $\lambda$, the isomorphism

$$
*_{\lambda}: \Gamma\left(\wedge^{\bullet} T M\right) \rightarrow \Gamma\left(\wedge^{n-\bullet} T^{*} M\right)
$$

is defined by $*_{\lambda} u=\iota_{u} \lambda$, for $u \in \Gamma\left(\wedge^{\bullet} T M\right)$.

The modular vector field $X_{\lambda}$ is related to the $(n-1)$-form, $\partial_{\pi} \lambda$, by

$$
{ }_{\lambda} X_{\lambda}=-\partial_{\pi} \lambda \text {. }
$$

- Let us consider the operator on $\Gamma\left(\wedge^{\bullet} T M\right)$, of degree -1 ,

$$
\partial_{\lambda}=-\left(*_{\lambda}\right)^{-1} \circ \mathrm{d} \circ *_{\lambda} .
$$


On vector fields, this operator coincides with $-\operatorname{div}_{\lambda}$. Applied to the Poisson bivector, it yields the modular vector field,

$$
X_{\lambda}=\partial_{\lambda} \pi .
$$

Thus the modular vector field $X_{\lambda}$ can be considered as the "divergence" of the Poisson bivector $\pi$.

- Let us also consider the operator on $\Gamma\left(\wedge^{\bullet} T^{*} M\right)$, of degree -1 ,

$$
\partial_{\pi, \lambda}=-*_{\lambda} \circ \mathrm{d}_{\pi} \circ\left(*_{\lambda}\right)^{-1} .
$$

Both operators $\partial_{\pi}$ and $\partial_{\pi, \lambda}$ are generators of square zero of the Gerstenhaber algebra $\left(\Gamma\left(\wedge^{\bullet} T^{*} M\right),[,]_{\pi}\right)$. In view of equation (1.1), the difference $\partial_{\pi, \lambda}-\partial_{\pi}$ is the interior product by a vector field. In fact, this vector field is a 1-cocycle in the $\mathrm{d}_{\pi}$-cohomology $[80,125,68]$, and it coincides with the modular vector field,

$$
\partial_{\pi, \lambda}-\partial_{\pi}=\iota_{X_{\lambda}}
$$

\section{The modular class of a Lie algebroid with a Poisson structure}

It is straightforward to generalize the definition of the modular class from the case of the Poisson manifolds to that of the Lie algebroids with a Poisson structure. Let $\left(A, a_{A},[,]_{A}\right)$ be a Lie algebroid with a Poisson bivector $\pi \in \Gamma\left(\wedge^{2} A\right)$. Since the exact 1-forms, $\mathrm{d}_{A} f, f \in C^{\infty}(M)$, do not, in general, span the space of sections of $A^{*}$, we cannot rely on the original definition (2.1), but, assuming that $A$ is orientable, we choose a nowhere-vanishing section $\lambda$ of $\wedge^{\operatorname{top}}\left(A^{*}\right)$, and we consider the expressions in formulas (2.2)-(2.5), where $\mathrm{d}$ is replaced by $\mathrm{d}_{A}, \mathcal{L}$ by $\mathcal{L}^{A}$, where $\mathcal{L}_{X}^{A}=\left[\mathrm{d}_{A}, \iota_{X}\right]$ for $X \in \Gamma A, \partial_{\lambda}$ by $-\left(*_{\lambda}\right)^{-1} \circ \mathrm{d}_{A} \circ *_{\lambda}$, etc. Each of the equations (2.2)-(2.5) defines uniquely the same section $X_{\lambda}$ of $A$ which is a 1-cocycle in the $\mathrm{d}_{\pi}$-cohomology. Furthermore, the class of $X_{\lambda}$ is independent of the choice of section of $\wedge^{\text {top }}\left(A^{*}\right)$. The case of a Poisson manifold $M$ is recovered when $A=T M$.

Definition 2. The section $X_{\lambda}$ of $A$ is called a modular section of the Lie algebroid with a Poisson structure $(A, \pi)$. The $\mathrm{d}_{\pi}$-cohomology class of $X_{\lambda}$ is called the modular class of $(A, \pi)$.

We shall denote the modular class of $(A, \pi)$ by $\theta(A, \pi)$. When $A=T M$, the modular class of $(A, \pi)$ is the modular class $\theta(\pi)$ of the Poisson manifold $(M, \pi)$.

The non-orientable case can be dealt with by using densities instead of volume forms.

In the next section, we shall define the modular class, $\operatorname{Mod} E$, of a Lie algebroid $E$, and in Section 5.2 we shall show how $\theta(A, \pi)$ is related to $\operatorname{Mod}\left(A^{*}\right)$ when $A^{*}$ is the Lie algebroid with anchor $a_{A} \circ \pi^{\sharp}$ and bracket $[,]_{\pi}$.

\section{The modular class of a Lie algebroid}

The modular class of a Lie algebroid was introduced by Weinstein in [124]. This section summarizes some of the results of [34], in which the theory was developed.

\subsection{Lie algebroid representations}

A representation $[93,94]$ of a Lie algebroid $\left(E, a_{E},[,]_{E}\right)$ with base $M$ in a vector bundle $V$ with base $M$ is a map $D: \Gamma E \times \Gamma V \rightarrow \Gamma V,(u, s) \mapsto D_{u} s$, such that

$$
\begin{aligned}
& D_{f u} s=f D_{u} s, \\
& D_{u}(f s)=f D_{u} s+\left(a_{E}(u) \cdot f\right) s, \\
& D_{[u, v]_{E}}=\left[D_{u}, D_{v}\right],
\end{aligned}
$$


for all $u, v \in \Gamma E, s \in \Gamma V$ and $f \in C^{\infty}(M)$. A representation of $E$ in $V$ is also called a flat E-connection [125] on $V$, and $V$ is called an $E$-module $[117,36]$ if there is a representation of $E$ on $V$. Clearly, if $E=T M$, a flat $E$-connection is a flat connection in the usual sense, and if $M=\{\mathrm{pt}\}$, this notion reduces to the Lie algebra representations in vector spaces.

The canonical representation [34] of a Lie algebroid $E$ is the representation $D^{E}$ of $E$ in the line bundle $L^{E}=\wedge^{\text {top }} E \otimes \wedge^{\text {top }}\left(T^{*} M\right)$ defined by

$$
D_{u}^{E}(\lambda \otimes \nu)=\mathcal{L}_{u}^{E} \lambda \otimes \nu+\lambda \otimes \mathcal{L}_{a_{E}(u)} \nu,
$$

where $u \in \Gamma E, \lambda \in \Gamma\left(\wedge^{\text {top }} E\right), \nu \in \Gamma\left(\wedge^{\text {top }} T^{*} M\right)$, and where $\mathcal{L}_{u}^{E} \lambda=[u, \lambda]_{E}$.

When $E$ is the integrable sub-bundle of $T M$ defining a foliation $\mathcal{F}$ of $M$, the line bundle $L^{E}$ is isomorphic to the top exterior power of the conormal bundle to $\mathcal{F}$, and the representation $D^{E}$ is the top exterior power of the Bott connection of $\mathcal{F}$. When the normal bundle is orientable, the sections of $L^{E}$ are the tranverse measures to the foliation.

\subsection{Characteristic class of a Lie algebroid representation in a line bundle}

Let $E$ be a Lie algebroid with a representation $D$ in a line bundle $L$, and let $s$ be a nowherevanishing section of $L$. Define $\theta_{s} \in \Gamma\left(E^{*}\right)$ by the condition

$$
\left\langle\theta_{s}, u\right\rangle s=D_{u} s
$$

for all $u \in \Gamma E$. Then $\theta_{s}$ is a $\mathrm{d}_{E^{-}}$cocycle. Furthermore, the class of $\theta_{s}$ is independent of the choice of section $s$ of $L$, whence the following definition [34].

Definition 3. The section $\theta_{s}$ of $E^{*}$ is called a characteristic cocycle associated with the representation $D$ and the section $s$. Its class is called the characteristic class of the representation $D$.

If $L$ is not trivial, the class of $D$ is defined as one-half that of the class of the associated representation of $E$ in the square of $L$.

\subsection{The modular class of a Lie algebroid}

Equation (4.1) defines the canonical representation of the Lie algebroid $E$ in the line bundle, $L^{E}=\wedge^{\text {top }} E \otimes \wedge^{\text {top }} T^{*} M$, which appears in the following definition [34].

Definition 4. The characteristic class of the canonical representation $D^{E}$ of $E$ is called the modular class of the Lie algebroid $E$. A cocycle belonging to the modular class of $E$ is called a modular cocycle of $E$.

We shall denote the modular class of $E$ by $\operatorname{Mod} E$. Thus, by definition, in the orientable case, Mod $E$ is the $\mathrm{d}_{E}$-class of the 1-cocycle $\theta \in \Gamma\left(E^{*}\right)$, depending on the nowhere-vanishing section $\lambda \otimes \nu$ of $L^{E}$, such that

$$
\langle\theta, u\rangle \lambda \otimes \nu=\mathcal{L}_{u}^{E} \lambda \otimes \nu+\lambda \otimes \mathcal{L}_{a_{E}(u)} \nu,
$$

for all $u \in \Gamma E$.

Example 6. If $E=T M$, the modular class vanishes, $\operatorname{Mod}(T M)=0$, since one can choose $\lambda \in \Gamma\left(\wedge^{\text {top }} T^{*} M\right)$ and $\nu \in \Gamma\left(\wedge^{\text {top }} T M\right)$ such that $\langle\lambda, \nu\rangle=1$.

If $E$ is a Lie algebra $\mathfrak{g}$, then Mod $\mathfrak{g}$ is the infinitesimal modular character, $\operatorname{Tr}(\mathrm{ad})$, of $\mathfrak{g}$. Comparing this fact with the result in Section 2.1, we see that the modular class of $\mathfrak{g}$, considered as a Lie algebroid over a point, is equal to the modular class of the Poisson manifold $\mathfrak{g}^{*}$. 
Example 7. If $\mathfrak{g}$ is the non-Abelian 2-dimensional Lie algebra with commutation relation $\left[e_{1}, e_{2}\right]=e_{2}$, then $\operatorname{Mod} \mathfrak{g}=e_{1}^{*}$. We saw in Example 5 of Section 2.1 that $e_{1}^{*}$ is a modular vector field of the Poisson manifold $\mathfrak{g}^{*}$.

The preceding property of Lie algebras can be extended to Lie algebroids in the following way. Let us identify each section of a vector bundle $F \rightarrow M$ with its vertical lift, which is a section of the vertical tangent bundle $V F \rightarrow F$. This vertical lift, in turn, can be identified with a vector field on $F$, tangent to the fibers and invariant by translations along each fiber. In this way, the modular class of a Lie algebroid $E$ can be identified $[124,103]$ with the modular class of the Poisson manifold $E^{*}$, when $E^{*}$ is equipped with the linear Poisson structure (see, e.g., [94]) defined by the anchor and bracket of $E$.

Example 8. When $E$ is the Lie algebroid associated to a foliation $\mathcal{F}$, and the normal bundle of $\mathcal{F}$ is orientable, the sections of $L^{E}$ are the tranverse measures to the foliation. Therefore the modular class of $E$ is the obstruction to the existence of a transverse measure invariant under all vector fields tangent to the foliation.

Remark 1. A section of a vector bundle $E^{*}$ can be considered as a function on $E$ viewed as a supermanifold, and a nowhere-vanishing section of $L^{E}$ determines a Berezinian volume on the supermanifold $E$. A modular cocycle of a Lie algebroid $E$ is the divergence with respect to such a Berezinian volume of $\mathrm{d}_{E}$ considered as a vector field on the supermanifold $E$ (see [34]).

\subsection{The case of Poisson manifolds}

If $(M, \pi)$ is a Poisson manifold, the comparison of the modular class, $\theta(\pi)$, of $(M, \pi)$ with the modular class, Mod $\left(T^{*} M\right)$, of the Lie algebroid $\left(T^{*} M, \pi^{\sharp},[,]_{\pi}\right)$ yields the following result which was proved in [34].

Theorem 1. For a Poisson manifold, $(M, \pi)$, the modular classes $\theta(\pi)$ and $\operatorname{Mod}\left(T^{*} M\right)$ are equal, up to a factor 2 ,

$$
\theta(\pi)=\frac{1}{2} \operatorname{Mod}\left(T^{*} M\right)
$$

If $A$ is a Lie algebroid with a Poisson structure defined by $\pi \in \Gamma\left(\wedge^{2} A\right)$, the question arises whether the cohomology class, $\theta(A, \pi)$, that was defined in Section 3 , and $\operatorname{Mod}\left(\mathrm{A}^{*}\right)$, the modular class of the Lie algebroid $\left(A^{*}, a_{A} \circ \pi^{\sharp},[,]_{\pi}\right)$ satisfy a relation as simple as (4.2). The answer is no in general, and the correct relation is obtained by the introduction of a new notion, the modular class of a morphism.

\section{The modular class of a Lie algebroid morphism}

This section deals first with the modular classes of base-preserving Lie algebroid morphisms which were introduced in [45] and [77]. In Section 5.4, we shall show how the definition and properties of these classes can be extended to general morphisms.

\section{$5.1 \quad$ Lie algebroid morphisms}

By definition, if $E$ and $F$ are Lie algebroids over the same base, a vector bundle map $\Phi: E \rightarrow F$ is a Lie algebroid morphism (over the identity of $M$ ) if $a_{E}=a_{F} \circ \Phi$ and $\Phi$ induces a Lie algebra homomorphism from $\Gamma E$ to $\Gamma F$. It is well known (see, e.g., $[74,53])$ that $\Phi$ is a morphism if and only if $\wedge^{\bullet}\left(\Phi^{*}\right)$ defines a chain map from $\left(\Gamma\left(\wedge^{\bullet} F^{*}\right), \mathrm{d}_{F}\right)$ to $\left(\Gamma\left(\wedge^{\bullet} E^{*}\right), \mathrm{d}_{E}\right)$. 
Given a morphism $\Phi: E \rightarrow F$, let us consider the well-defined class in the Lie algebroid cohomology of $E$,

$$
\operatorname{Mod}(\Phi)=\operatorname{Mod} E-\Phi^{*}(\operatorname{Mod} F)
$$

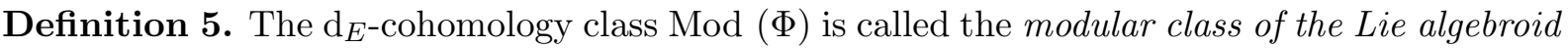
morphism $\Phi$.

The modular class of $\Phi$ can be considered as a relative modular class of the pair $(E, F)$, whence the terminology adopted in [77]. A representative of the class $\operatorname{Mod}(\Phi)$ is a section of $E^{*}$. It is clear that the modular class of an isomorphism of Lie algebroids vanishes.

It was proved in [77] that the modular class of a morphism $\Phi: E \rightarrow F$ is the characteristic class of a representation of $E$. In fact, set $L^{E, F}=\wedge^{\text {top }} E \otimes \wedge^{\text {top }} F^{*}$ and define the map $D^{\Phi}$ by

$$
D_{u}^{\Phi}(\lambda \otimes \nu)=\mathcal{L}_{u}^{E} \lambda \otimes \nu+\lambda \otimes \mathcal{L}_{\Phi u}^{F} \nu
$$

for $u \in \Gamma E$ and $\lambda \otimes \nu \in \Gamma\left(L^{E, F}\right)$.

Proposition 6. When $\Phi: E \rightarrow F$ is a Lie algebroid morphism, the map $D^{\Phi}$ is a representation of $E$ on the line bundle $L^{E, F}$, and the modular class of $\Phi$ is the characteristic class of this representation.

\subsection{The modular class of a Lie algebroid with a Poisson structure revisited}

Let us again consider a Lie algebroid $A$ with a Poisson structure defined by $\pi \in \Gamma\left(\wedge^{2} A\right)$, as in Section 3. We can now establish a relation between the cohomology class $\theta(A, \pi)$ and $\operatorname{Mod}\left(\mathrm{A}^{*}\right)$, the modular class of the Lie algebroid $\left(A^{*}, a_{A} \circ \pi^{\sharp},[,]_{\pi}\right)$. (The modular class $\theta(A, \pi)$ is the class of a section of $A$, not to be confused with the modular class of $A$ as a Lie algebroid, $\operatorname{Mod} A$, which is the class of a section of $A^{*}$.)

Theorem 2. The modular class of a Lie algebroid with Poisson structure, $(A, \pi)$, and the modular class of the morphism $\pi^{\sharp}: A^{*} \rightarrow A$ are equal up to a factor 2 ,

$$
\theta(A, \pi)=\frac{1}{2} \operatorname{Mod}\left(\pi^{\sharp}\right) .
$$

The equality (5.2) yields the desired relation between $\theta(A, \pi)$ and $\operatorname{Mod}\left(\mathrm{A}^{*}\right)$,

$$
\theta(A, \pi)=\frac{1}{2}\left(\operatorname{Mod}\left(A^{*}\right)-\left(\pi^{\sharp}\right)^{*}(\operatorname{Mod} A)\right) .
$$

In particular, if $\pi$ is non-degenerate, $\theta(A, \pi)$ vanishes.

In the case of a Poisson manifold, $A=T M$. Since $\operatorname{Mod}(T M)=0,(5.3)$ reduces in this case to $\theta(\pi)=\frac{1}{2} \operatorname{Mod}\left(T^{*} M\right)$, i.e., we recover (4.2), the result of Theorem 1 .

If $A=\mathfrak{g}$ is a Lie algebra equipped with a solution $\pi=r$ of the classical Yang-Baxter equation (1.7), then $(\mathfrak{g}, r)$ can be considered as a Lie algebroid over a point with a Poisson structure, and as such it has a modular class, $\theta(\mathfrak{g}, r)$.

Example 9. Let $\mathfrak{g}$ be the 2-dimensional non-Abelian Lie algebra as in Example 5 of Section 2.1. Then $r=e_{1} \wedge e_{2}$ is a solution of the classical Yang-Baxter equation on $\mathfrak{g}$. Because $r$ is nondegenerate, $\theta(\mathfrak{g}, r)=0$. On the other hand, the modular class of the Lie algebra $\mathfrak{g}^{*}$ equipped with the Lie bracket $[,]_{r}$ defined by (1.8) can be computed as the trace of the adjoint representation of this Lie algebra, yielding $\operatorname{Mod}\left(\mathfrak{g}^{*}\right)=-e_{2}$. Since $\operatorname{Mod} \mathfrak{g}=e_{1}^{*}$, we again obtain $\theta(\mathfrak{g}, r)=\frac{1}{2}\left(\operatorname{Mod}\left(\mathfrak{g}^{*}\right)+r^{\sharp}(\operatorname{Mod} \mathfrak{g})\right)=\frac{1}{2}\left(-e_{2}+r^{\sharp} e_{1}^{*}\right)=0$. 


\subsection{Unimodularity}

A Lie algebroid or a Lie algebroid morphism is called unimodular if its modular class vanishes. Examples of unimodular Lie algebroids are tangent bundles and unimodular Lie algebras, and we have mentioned above in Example 8 of Section 4.3 the meaning of unimodularity in the theory of foliations. The unimodularity of morphisms also is related to the existence of invariant transverse measures. For example, if $H$ is a connected, closed subgroup of a connected Lie group $G$, with Lie algebras $\mathfrak{h} \subset \mathfrak{g}$, the canonical injection $i: \mathfrak{h} \hookrightarrow \mathfrak{g}$ is unimodular if and only if there exists a $G$-invariant measure on the homogeneous space $G / H[77]$.

Remark 2. When viewed as a supermanifold, an orientable unimodular Lie algebroid is a QSmanifold in the sense of Schwarz [112] (see [76]).

A Poisson structure, $\pi$, on a Lie algebroid, $A$, is also called unimodular if the class $\theta(A, \pi)$ vanishes. A Poisson manifold $(M, \pi)$ is unimodular if $\theta(\pi)=0$, i.e., if there exists a vanishing modular vector field $X_{\lambda}$, i.e., if there exists a density that is invariant under all Hamiltonian vector fields.

On an orientable Lie algebroid with a Poisson structure, the Poisson cohomology $H^{\bullet}\left(A, \mathrm{~d}_{\pi}\right)$ and the Poisson homology $H_{\bullet}\left(A, \partial_{\pi}\right)$ can be compared. When $\lambda$ is a volume element on $A, *_{\lambda}$ is a chain map from the complex $\left(\Gamma\left(\wedge^{\bullet} A^{*}\right), \partial_{\pi}\right)$ to the complex $\left(\Gamma\left(\wedge^{n-\bullet} A\right), \mathrm{d}_{\pi}+\epsilon_{X_{\lambda}}\right)$, where $n$ is the rank of $A$ and $\epsilon_{X}$ is the exterior product of multivectors by the section $X$ of $A$. In fact, in view of equation (2.5), $\partial_{\pi}=\partial_{\pi, \lambda}-\iota_{X_{\lambda}}=-*_{\lambda} \circ\left(\mathrm{d}_{\pi}+\epsilon_{X_{\lambda}}\right) \circ\left(*_{\lambda}\right)^{-1}$. Therefore, the homology of the complex $\left(\Gamma\left(\wedge^{\bullet} A^{*}\right), \partial_{\pi}\right)$ is isomorphic to the cohomology of the complex $\left(\Gamma\left(\wedge^{\bullet} A\right), \mathrm{d}_{\pi}+\epsilon_{X_{\lambda}}\right)$ $[54,55,125,68]$. In particular,

Proposition 7. When the Poisson structure, $\pi$, of an orientable Lie algebroid, $A$, is unimodular, its Poisson homology and Poisson cohomology are isomorphic,

$$
H_{\bullet}\left(A, \partial_{\pi}\right) \simeq H^{\mathrm{top}-\bullet}\left(A, \mathrm{~d}_{\pi}\right) .
$$

Remark 3. More general pairings between Lie algebroid cohomology and homology are to be found in [55, 34, 125]. In [45], Grabowski, Marmo and Michor use odd volume forms to define generating operators of square zero of the Gerstenhaber algebra $\Gamma\left(\wedge^{\bullet} A\right)$ for an arbitrary Lie algebroid $A$, and they show that the associated homology is independent of the choice of odd volume form and that, when the Lie algebroid is orientable, there is a Poincaré duality between this homology and the Lie algebroid cohomology.

\subsection{General morphisms of Lie algebroids}

The extension of the definition and properties of the modular class to the case of Lie algebroid morphisms that are not necessarily base-preserving is the subject of the article [73]. Let us briefly review this general case. When $\Phi$ is a vector bundle map from $E \rightarrow M$ to $F \rightarrow N$ over a map $\phi: M \rightarrow N$, there is a map $\widetilde{\Phi}^{*}$ from the sections of $F^{*} \rightarrow N$ to the sections of $E^{*} \rightarrow M$, induced from the base-preserving vector bundle morphism $\Phi^{*}: \phi^{!} F^{*} \rightarrow E^{*}$, where $\phi^{!} F^{*}$ is the pull-back of $F^{*}$ under $\phi$. Then $\widetilde{\Phi}^{*}$ is extended as an exterior algebra homomorphism $\wedge^{\bullet} \widetilde{\Phi}^{*}: \Gamma\left(\wedge^{\bullet} F^{*}\right) \rightarrow \Gamma\left(\wedge^{\bullet} E^{*}\right)$. Let $E \rightarrow M$ and $F \rightarrow N$ be Lie algebroids. By definition, $\Phi$ is a Lie algebroid morphism if $\wedge^{\bullet} \widetilde{\Phi}^{*}$ is a chain map from $\left(\Gamma\left(\wedge^{\bullet} F^{*}\right), \mathrm{d}_{F}\right)$ to $\left(\Gamma\left(\wedge^{\bullet} E^{*}\right), \mathrm{d}_{E}\right)$.

Remark 4. It was proved by Chen and Liu [20] that this definition is equivalent to the original definition of morphism in [49, 93, 94]. In fact, it is also equivalent to the definition in [117]: $\Phi$ maps the homological vector field $\mathrm{d}_{E}$ on the supermanifold $E$ to $\mathrm{d}_{F}$. 


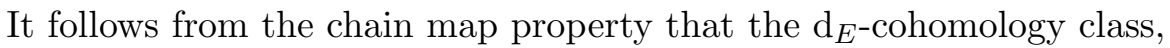

$$
\operatorname{Mod}(\Phi)=\operatorname{Mod} E-\widetilde{\Phi}^{*}(\operatorname{Mod} F)
$$

is well-defined. It is called the modular class of the morphism $\Phi$, a definition that generalizes Definition 5.

That this class is the characteristic class of a representation of $E$ is proved using the nontrivial fact that the pull-back of a Lie algebroid representation by a morphism exists. Another theorem in [73] states that the pull-back of a Lie algebroid by a transverse map, in particular by a submersion, gives rise to a morphism with vanishing modular class.

\section{The regular case and triangular Lie bialgebras}

A Lie algebroid $A$ over $M$ is called regular if the image of its anchor map is of constant rank, and thus a sub-bundle of $T M$. The Lie algebroids associated to foliations are an important example of regular Lie algebroids. The modular classes of regular Lie algebroids were considered in $[24,35,36]$.

A Poisson manifold $(M, \pi)$ is called regular if its cotangent Lie algebroid $T^{*} M$ is regular, i.e., if the image of the map $\pi^{\sharp}: T^{*} M \rightarrow T M$ is of constant rank. By extension, a Poisson structure, $\pi$, on a Lie algebroid, $A$, is called regular if the image of $\pi^{\sharp}$ is of constant rank, and thus a Lie subalgebroid of $A$. We now summarize results on the modular class of Lie algebroids with a regular Poisson structure obtained in [78].

\subsection{The regular case}

Let $(A, \pi)$ be a Lie algebroid with a regular Poisson structure. Let $B=\pi^{\sharp}\left(A^{*}\right)$ and let us denote the Lie algebroid morphism $\pi^{\sharp}$ considered as a submersion onto $B$ by $\pi_{B}^{\sharp}$. There is an exact sequence of Lie algebroids over the same base,

$$
0 \rightarrow \operatorname{Ker} \pi^{\sharp} \rightarrow A^{*} \stackrel{\pi_{B}^{\sharp}}{\longrightarrow} B \rightarrow 0 .
$$

The canonical representation of $B$ on Ker $\pi^{\sharp}$ is obtained by factoring the adjoint action of $\Gamma\left(A^{*}\right)$, with respect to the Lie bracket $[,]_{\pi}$ defined by (1.2), on $\Gamma\left(\operatorname{Ker} \pi^{\sharp}\right)$ through the submersion $\pi_{B}^{\sharp}: A^{*} \rightarrow B$. (The existence of this representation follows from [94], Proposition 3.3.20.) Explicitly,

$$
X \cdot \gamma=\mathcal{L}_{X}^{A} \gamma
$$

for $X \in \Gamma B, \gamma \in \Gamma\left(\operatorname{Ker} \pi^{\sharp}\right)$.

Theorem 3. The modular class, $\theta(A, \pi)$, of a Lie algebroid with a regular Poisson structure, $(A, \pi)$, satisfies

$$
\theta(A, \pi)=\left(\pi_{B}^{\sharp}\right)^{*}\left(\theta_{B}\right),
$$

where $\theta_{B}$ is the characteristic class of the representation of $B$ in $\wedge^{\operatorname{top}}\left(\operatorname{Ker} \pi^{\sharp}\right)$ induced from the canonical representation.

This theorem permits an efficient computation of a representative of the modular class, avoiding the computation of terms which mutually cancel in equation (5.3).

Remark 5. Formula (6.1) can also be obtained from the more general computation of the modular class of a Lie algebroid morphism with constant rank and unimodular kernel in [73]. 


\subsection{Triangular Lie bialgebras}

This section summarizes some of the results of [78] concerning finite-dimensional real or complex Lie algebras with a triangular $r$-matrix, the definition of which was recalled in Section 1.6.

Let $\mathfrak{g}$ be a Lie algebra considered as a Lie algebroid over a point, and let $r \in \wedge^{2} \mathfrak{g}$ be a triangular $r$-matrix on $\mathfrak{g}$, defining a triangular Lie bialgebra structure on $\left(\mathfrak{g}, \mathfrak{g}^{*}\right)$. The image of $r^{\sharp}: \mathfrak{g}^{*} \rightarrow \mathfrak{g}$ is called the carrier of $r$. We set $\mathfrak{p}=\operatorname{Im} r^{\sharp}$. In this case, the canonical representation of $\mathfrak{p}$ on Ker $r^{\sharp}$ coincides with the restriction of the coadjoint representation of $\mathfrak{g}$, and it is dual to the representation of $\mathfrak{p}$ on $\mathfrak{g} / \mathfrak{p}$ induced from the adjoint action of $\mathfrak{g}$. Therefore, as a conseqeunce of Theorem 3, we obtain the following proposition, where $\chi_{\mathfrak{p}, V}$ denotes the infinitesimal character of a representation $V$ of Lie algebra $\mathfrak{p}$, and $r_{(\mathfrak{p})}^{\sharp}$ denotes $r^{\sharp}$ considered as a skew-symmetric isomorphism from $\mathfrak{p}^{*}=\mathfrak{g}^{*} / \operatorname{Ker} r^{\sharp}$ to $\mathfrak{p}$.

Proposition 8. Let $r$ be a triangular $r$-matrix on a Lie algebra $\mathfrak{g}$. The modular class of $(\mathfrak{g}, r)$ is the element $\theta(\mathfrak{g}, r)$ of $\mathfrak{g}$ such that

$$
\theta(\mathfrak{g}, r)=-r_{(\mathfrak{p})}^{\sharp}\left(\chi_{\mathfrak{p}, \operatorname{Ker}\left(r^{\sharp}\right)}\right)=r_{(\mathfrak{p})}^{\sharp}\left(\chi_{\mathfrak{p}, \mathfrak{g} / \mathfrak{p})}\right) .
$$

\subsection{Frobenius Lie algebras}

The following proposition was proved by Stolin [114] (see also [42, 52]).

Proposition 9. Let $\omega \in \wedge^{2}\left(\mathfrak{p}^{*}\right)$ be a non-degenerate 2 -form on a Lie algebra $\mathfrak{p}$, and let $r \in \wedge^{2} \mathfrak{p}$ be the inverse of $\omega$. Then $\mathrm{d}_{\mathfrak{p}} \omega=0$ if and only if $[r, r]_{\mathfrak{g}}=0$.

A Lie algebra $\mathfrak{p}$ is called a quasi-Frobenius (resp., Frobenius) Lie algebra if there exists a nondegenerate 2-cocyle (resp., 2-coboundary) on $\mathfrak{p}$. A Lie algebra $\mathfrak{p}$ is called Frobenius with respect to $\xi \in \mathfrak{p}^{*}$ if $\omega=-\mathrm{d}_{\mathfrak{p}} \xi$ is a non-degenerate 2 -form.

From this definition and from Proposition 9, we obtain:

Proposition 10. Let $\mathfrak{g}$ be a Lie algebra, and let $\mathfrak{p}$ be a Lie subalgebra of $\mathfrak{g}$. Assume that $\mathfrak{p}$ is Frobenius with respect to $\xi \in \mathfrak{p}^{*}$. Then $r=-\left(\mathrm{d}_{\mathfrak{p}} \xi\right)^{-1} \in \wedge^{2} \mathfrak{p}$ is a triangular $r$-matrix on $\mathfrak{g}$, i.e., a solution of the classical Yang-Baxter equation.

When the carrier of a triangular $r$-matrix is a Frobenius Lie algebra, Proposition 8 yields the following result.

Theorem 4. Let $r$ be a triangular $r$-matrix on a Lie algebra $\mathfrak{g}$. Assume that $\mathfrak{p}=\operatorname{Im} r^{\sharp}$ is Frobenius with respect to $\xi \in \mathfrak{p}^{*}$. Then $\theta(\mathfrak{g}, r)$ is the unique element $X \in \mathfrak{g}$ such that

$$
\operatorname{ad}_{X}^{*} \xi=\chi_{\mathfrak{p}, \mathfrak{g} / \mathfrak{p}}
$$

Example 10. We illustrate this theorem with the example where $\mathfrak{p} \subset \mathfrak{s l}_{3}(\mathbb{R})$ is the Lie algebra of traceless matrices of the form $\left(\begin{array}{ccc}\bullet & \bullet & \bullet \\ 0 & \bullet & \bullet \\ 0 & \bullet & \bullet\end{array}\right)$. It is Frobenius with respect to $\xi=e_{12}^{*}+e_{23}^{*}$.

The coboundary of $-\xi$ is $-\mathrm{d}_{\mathfrak{p}} \xi=2\left(e_{11}^{*} \wedge e_{12}^{*}+e_{12}^{*} \wedge e_{22}^{*}+e_{13}^{*} \wedge e_{32}^{*}+e_{22}^{*} \wedge e_{23}^{*}+e_{23}^{*} \wedge e_{33}^{*}\right)$, and the corresponding triangular $r$-matrix is

$$
r_{G G}=\left(\frac{2}{3} e_{11}-\frac{1}{3}\left(e_{22}+e_{33}\right)\right) \wedge e_{12}+\left(\frac{1}{3}\left(e_{11}+e_{22}\right)-\frac{2}{3} e_{33}\right) \wedge e_{23}+e_{13} \wedge e_{32} .
$$

The image of $r_{G G}^{\sharp}$ is $\mathfrak{p}$ and its kernel is generated by $e_{21}^{*}$ and $e_{31}^{*}$. Computing the character $\chi_{\mathfrak{p}, \mathfrak{g} / \mathfrak{p}}=-2 e_{11}^{*}+e_{22}^{*}+e_{33}^{*}$, and solving equation (6.2), we obtain $\theta\left(\mathfrak{s l}_{3}(\mathbb{R}), r_{G G}\right)=-2 e_{12}+e_{23}$. The $r$-matrix $r_{G G}$ is the Gerstenhaber-Giaquinto generalized Jordanian $r$-matrix [42] on $\mathfrak{s l}_{3}(\mathbb{R})$. See [78] for the computation of the modular class in the general case of $\mathfrak{s l}_{n}(\mathbb{R})$. 


\section{The modular class of a twisted Poisson structure}

Twisted Poisson structures on manifolds were studied by Ševera and Weinstein [113] and such structures on Lie algebroids were subsequently defined by Roytenberg in [111]. It was proved there that a twisted Poisson structure on $A$ corresponds to a quasi-Lie-bialgebroid structure on $\left(A, A^{*}\right)$, generalizing the triangular Lie bialgebroids defined by Poisson structures. The closed 3 -form which we denote by $\psi$ below, to conform with the notation in [63] and subsequent publications, plays an important role in generalized complex geometry [48] and, consequently, in recent studies on sigma-models, where it is usually denoted by $H$, and sometimes called an $H$-flux.

\subsection{Twisted Poisson structures}

A twisted Poisson structure on a Lie algebroid $A$ is a pair $(\pi, \psi)$, where $\pi \in \Gamma\left(\wedge^{2} A\right)$ and $\psi$ is a $d_{A \text {-closed form on } A \text { satisfying }}$

$$
\frac{1}{2}[\pi, \pi]_{A}=\left(\wedge^{3} \pi^{\sharp}\right) \psi .
$$

When a twisted Poisson structure is defined on the Lie algebroid $T M$, the manifold $M$ is called a twisted Poisson manifold.

Example 11. There is no genuine twisted Poisson structure on a Poisson manifold of dimension $\leq 3$, since the image of $\pi^{\sharp}$ is then of dimension at most 2 . We give an example [50] of a twisted Poisson structure on an open subset of $\mathbb{R}^{4}, M=\left\{\left(x_{1}, x_{2}, x_{3}, x_{4}\right) \in \mathbb{R}^{4} \mid x_{1} \neq 0\right.$ and $\left.x_{3} \neq 0\right\}$. Denote $\frac{\partial}{\partial x_{i}}$ by $\partial_{i}$. Let $\pi=x_{3} \partial_{1} \wedge \partial_{2}+x_{1} \partial_{3} \wedge \partial_{4}$ and $\psi=\left(\left(x_{1}\right)^{-2} \mathrm{~d} x_{4}-\left(x_{3}\right)^{-2} \mathrm{~d} x_{2}\right) \wedge \mathrm{d} x_{1} \wedge \mathrm{d} x_{3}$. Then $(\pi, \psi)$ is a twisted Poisson structure on the manifold $M$.

When $(A, \pi, \psi)$ is a Lie algebroid with a twisted Poisson structure, the bracket defined by

$$
[\alpha, \beta]_{\pi, \psi}=\mathcal{L}_{\pi^{\sharp} \alpha}^{A} \beta-\mathcal{L}_{\pi^{\sharp} \beta}^{A} \alpha-\mathrm{d}_{A}(\pi(\alpha, \beta))+\psi\left(\pi^{\sharp} \alpha, \pi^{\sharp} \beta, \cdot\right),
$$

for $\alpha$ and $\beta \in \Gamma\left(A^{*}\right)$, satisfies the Jacobi identity. With the anchor $a_{A} \circ \pi^{\sharp}$ and the bracket $[,]_{\pi, \psi}, A^{*}$ is a Lie algebroid, and the map $\pi^{\sharp}$ is a Lie algebroid morphism from $A^{*}$ to $A$.

There is an associated Gerstenhaber bracket on $\Gamma\left(\wedge^{\bullet} A^{*}\right)$, also denoted by $[,]_{\pi, \psi}$, and an associated differential on $\Gamma\left(\wedge^{\bullet} A\right), \mathrm{d}_{\pi, \psi}=[\pi, \cdot]_{A}+\underline{\mathrm{d}}$, where $\underline{\mathrm{d}}$ is the interior product of sections of $\wedge^{\bullet} A$ by the bivector-valued 1 -form, $(\alpha, \beta) \mapsto \psi\left(\pi^{\sharp} \alpha, \pi^{\sharp} \beta, \cdot\right)$.

Remark 6. The pair $\left(A, A^{*}\right)$ is not in general a Lie bialgebroid. However, when $A$ is equipped with the bracket defined by $[X, Y]_{A, \psi}=[X, Y]_{A}+\psi\left(\pi^{\sharp}(\cdot), X, Y\right)$, for $X$ and $Y \in \Gamma A$, the pair $\left(A, A^{*}\right)$ becomes a quasi-Lie-bialgebroid. In terms of the big bracket [111, 71],

$$
\begin{aligned}
& {[X, Y]_{A, \psi}=\left\{\left\{X, \mu_{\psi}\right\}, Y\right\}, \quad \text { where } \quad \mu_{\psi}=\mu-\{\psi, \pi\}} \\
& {[\alpha, \beta]_{\pi, \psi}=\left\{\left\{\alpha, \gamma_{\psi}\right\}, \beta\right\}, \quad \text { where } \quad \gamma_{\psi}=-\{\mu, \pi\}+\frac{1}{2}\{\{\psi, \pi\}, \pi\} .}
\end{aligned}
$$

\subsection{The modular class in the twisted case}

The modular class of a twisted Poisson structure $(A, \pi, \psi)$ was first defined in [72] as the $d_{\pi, \psi^{-}}$ cohomology class of a section of $A$ depending on the choice of a volume form (for the case where $A=T M$, see also [7]). Then in [77], it was shown to be the modular class of the morphism $\pi^{\sharp}: A^{*} \rightarrow A$, up to a factor 2 . Here we proceed somewhat differently. 
Definition 6. The modular class, $\theta(A, \pi, \psi)$, of the twisted Poisson structure $(\pi, \psi)$ on $A$ is

$$
\theta(A, \pi, \psi)=\frac{1}{2} \operatorname{Mod}\left(\pi^{\sharp}\right) .
$$

A $d_{\pi, \psi^{-}}$cocycle in the modular class $\theta(A, \pi, \psi)$ is called a modular section of $(A, \pi, \psi)$.

Thus, we take (5.2) to be the definition of the modular class, and it is clear, because of Theorem 2, that Definition 6 generalizes Definition 2.

Example 12. When $\pi^{\sharp}: A^{*} \rightarrow A$ is an isomorphism of vector bundles, the modular class vanishes. For instance, the modular class of the twisted Poisson structure $(\pi, \psi)$ of Example 11 of Section 7.1 above vanishes.

In the case where $A=T M$, the modular class of the morphism $\pi^{\sharp}$ is that of the Lie algebroid $T^{*} M$. Therefore, Theorem 1 remains valid in the twisted case: The modular class of a twisted Poisson manifold is equal to one-half of the modular class of its cotangent bundle Lie algebroid.

The problem that arises is to determine a section of $A$ which is a representative of the modular class thus defined. Assume that $A$ is orientable, and let $\lambda \in \Gamma\left(\wedge^{\text {top }} A^{*}\right)$ be a volume form on $A$. Define sections $X_{\lambda}$ and $Y$ of $A$ by

$$
*_{\lambda} X_{\lambda}=-\partial_{\pi} \lambda, \quad Y=\pi^{\sharp} \iota_{\pi} \psi,
$$

and set $Z_{\lambda}=X_{\lambda}+Y$. In [72], it was proved that $Z_{\lambda}$ satisfies the relation

$$
\partial_{\pi, \psi, \lambda}-\partial=\iota_{Z_{\lambda}},
$$

where $\partial_{\pi, \psi, \lambda}=-*_{\lambda} \circ \mathrm{d}_{\pi, \psi} \circ\left(*_{\lambda}\right)^{-1}$ and $\partial=\partial_{\pi}+\underline{\partial}+\iota_{Y}$, the operator $\underline{\partial}$ being the dual of $\underline{\mathrm{d}}$, and also that both operators $\partial_{\pi, \psi, \lambda}$ and $\partial$ are generators of square zero of the Gerstenhaber bracket

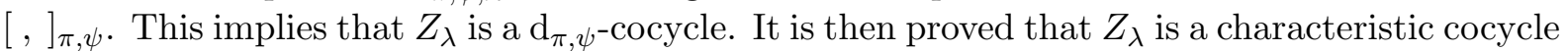
of the representation $D^{(\partial)}$ of $A^{*}$ on $\wedge^{\text {top }}\left(A^{*}\right)$ defined by $D_{\alpha}^{(\partial)}(\mu)=-\alpha \wedge \partial \mu$, for $\alpha \in \Gamma\left(A^{*}\right)$ and $\mu \in \Gamma\left(\wedge^{\text {top }} A^{*}\right)$. It was further remarked in [77] that, for $\Phi=\pi^{\sharp}$, the representation $D^{\Phi}$ defined by (5.1) is the square of the representation $D^{(\partial)}, D^{\pi^{\sharp}}(\mu \otimes \mu)=D^{(\partial)} \mu \otimes \mu+\mu \otimes D^{(\partial)} \mu$. Applying Proposition 6 to the morphism $\pi^{\sharp}$ proves the following theorem.

Theorem 5. The section $Z_{\lambda}=X_{\lambda}+Y$ of $A$ is a modular section of $(A, \pi, \psi)$.

We see that in the Poisson case, the modular section $Z_{\lambda}$ reduces to the modular section $X_{\lambda}$.

See [72] for the description of a unimodular twisted Poisson structure on a dense open set of a semi-simple Lie group. In this example the 3 -form $\psi$ is the Cartan 3 -form.

\subsection{The regular twisted case}

The formula for the modular class $\theta(A, \pi)$ of a regular Poisson structure given in Theorem 3 is valid for the modular class of a regular twisted Poisson structure [78]:

$$
\theta(A, \pi, \psi)=\left(\pi_{B}^{\sharp}\right)^{*}\left(\theta_{B}\right) .
$$

\subsection{Non-degenerate twisted Poisson structures}

It is well-known that the inverse of a non-degenerate Poisson structure, $\pi$, on a Lie algebroid is a symplectic structure, i.e., a non-degenerate 2-form, $\omega \in \Gamma\left(\wedge^{2} A^{*}\right)$. Recall that $\omega$ is defined by $\iota_{X}(\omega)=-\omega^{b}(X)$, for all $X \in \Gamma A$, where $\omega^{b}=\left(\pi^{\sharp}\right)^{-1}$. 
When a twisted Poisson structure $(A, \pi, \psi)$ is such that $\pi^{\sharp}$ is invertible, the inverse of $\pi$ is a 2 -form which is not $\mathrm{d}_{A}$-closed, but satisfies

$$
\mathrm{d}_{A} \omega=-\psi,
$$

and, conversely, equation (7.2) implies that $\left(\omega^{-1}, \psi\right)$ is a twisted Poisson structure. The nondegenerate 2 -form $\omega$ is then called twisted symplectic. (See [113, 78], and, for a generalization of this correspondence, see [71].) It is easy to construct non-degenerate twisted Poisson structures using this result.

Example 13. We shall show that Example 11 of Section 7.1 arises in this way, when $A$ is the tangent bundle of the manifold, $M$, and $\mathrm{d}_{A}$ is the de Rham differential, $\mathrm{d}$. We keep the notations of 7.1. Consider the non-degenerate 2-form on $M, \omega=\left(x_{3}\right)^{-1} \mathrm{~d} x_{1} \wedge \mathrm{d} x_{2}+\left(x_{1}\right)^{-1} \mathrm{~d} x_{3} \wedge \mathrm{d} x_{4}$. Then $-\mathrm{d} \omega=\left(\left(x_{1}\right)^{-2} \mathrm{~d} x_{4}-\left(x_{3}\right)^{-2} \mathrm{~d} x_{2}\right) \wedge \mathrm{d} x_{1} \wedge \mathrm{d} x_{3}$. The inverse of $\omega$ is the non-degenerate bivector $\pi=x_{3} \partial_{1} \wedge \partial_{2}+x_{1} \partial_{3} \wedge \partial_{4}$. So it follows without computations that the pair $(\pi, \psi)$, where $\psi=-\mathrm{d} \omega$, satisfies (7.1).

Let us consider a Lie algebroid, $A$, with a regular twisted Poisson structure, $(\pi, \psi)$, and let $B=\pi^{\sharp}\left(A^{*}\right)$ be the image of $\pi^{\sharp}$. The morphism $\pi^{\sharp}$ defines a skew-symmetric isomorphism from $B^{*}=A^{*} / \operatorname{Ker} \pi^{\sharp}$ to $B$. We denote the section of $\wedge^{2} B^{*}$ defined by the inverse of this isomorphism by $\omega_{(B)}$. If $\psi_{(B)}$ is the pull-back of $\psi$ to the sub-bundle $B$ of $A$, then $\mathrm{d}_{B}\left(\omega_{(B)}\right)=-\psi_{(B)}$. These considerations lead to the following proposition from [78] which describes a method for constructing twisted Poisson structures that uses the linearized form (7.2) of the twisted Poisson condition (7.1).

Proposition 11. Let $\omega$ be a 2-form on a Lie algebroid $A$ whose restriction $\left.\omega\right|_{B}$ to a Lie subalgebroid $B$ of $A$ is non-degenerate. Then the inverse of $\left.\omega\right|_{B}$ and $\psi=-\mathrm{d}_{A} \omega$ define a regular twisted Poisson structure on A.

The bivector $\pi$ of this twisted Poisson structure is such that $\pi^{\sharp}: A^{*} \rightarrow B$ is the composition of the canonical projection of $A^{*}$ onto $B^{*}$ with the inverse of $\left.\omega\right|_{B}$.

Example 14. On $M=\left\{\left(x_{1}, x_{2}, x_{3}, x_{4}, x_{5}\right) \in \mathbb{R}^{5} \mid x_{1} \neq 0\right.$ and $\left.x_{5} \neq 0\right\}$, consider the 2-form, $\omega=x_{5} \mathrm{~d} x_{1} \wedge \mathrm{d} x_{2}+x_{1} \mathrm{~d} x_{3} \wedge \mathrm{d} x_{4}$. The restriction of $\omega$ to the sub-bundle $B$ of $T M$ generated by $\partial_{1}, \partial_{2}, \partial_{3}$ and $\partial_{4}$ is non-degenerate, with inverse $\pi=\left(x_{5}\right)^{-1} \partial_{1} \wedge \partial_{2}+\left(x_{1}\right)^{-1} \partial_{3} \wedge \partial_{4}$. Then $(\pi,-\mathrm{d} \omega)$ is a twisted Poisson structure on $M$. Here $\mathrm{d} \omega=\mathrm{d} x_{1} \wedge \mathrm{d} x_{2} \wedge \mathrm{d} x_{5}+\mathrm{d} x_{1} \wedge \mathrm{d} x_{3} \wedge \mathrm{d} x_{4}$ and a computation yields $\frac{1}{2}[\pi, \pi]=-\left(\wedge^{3} \pi^{\sharp}\right)(\mathrm{d} \omega)=\left(x_{1}\right)^{-2}\left(x_{5}\right)^{-1} \partial_{2} \wedge \partial_{3} \wedge \partial_{4}$.

\subsection{Twisted triangular $r$-matrices}

When $A=\mathfrak{g}$ is a Lie algebra, and $\pi=r \in \wedge^{2} \mathfrak{g}$ and $\psi \in \wedge^{3}\left(\mathfrak{g}^{*}\right)$ satisfy relation $(7.1),(r, \psi)$ is called a twisted triangular structure, and $r$ is called a twisted triangular $r$-matrix. The formulas in Proposition 8 remain valid for the modular class of a Lie algebra with a twisted triangular $r$-matrix [78]:

$$
\theta(\mathfrak{g}, r, \psi)=-r_{(\mathfrak{p})}^{\sharp}\left(\chi_{\mathfrak{p}, \operatorname{Ker} r}^{\sharp}\right)=r_{(\mathfrak{p})}^{\sharp}\left(\chi_{\mathfrak{p}, \mathfrak{g} / \mathfrak{p}}\right) .
$$

Example 15. Examples of twisted triangular $r$-matrices are given in [78]. They can be constructed as indicated in Proposition 11. We quote the simplest example with a non-vanishing modular class. Let $\mathfrak{p}$ be the Lie subalgebra of $\mathfrak{g}=\mathfrak{g l}_{3}(\mathbb{R})$ with zeros on the third line, which is the Lie algebra of the group of affine transformations of $\mathbb{R}^{2}$. Consider the 2-cochain on the Lie algebra $\mathfrak{g}, \omega=e_{12}^{*} \wedge e_{21}^{*}+e_{11}^{*} \wedge e_{13}^{*}+e_{22}^{*} \wedge e_{23}^{*}$. The restriction of $\omega$ to $\mathfrak{p}$ is invertible and its inverse is $r=e_{12} \wedge e_{21}+e_{11} \wedge e_{13}+e_{22} \wedge e_{23}$. Then $(r, \psi)$, with $\psi=-\mathrm{d}_{\mathfrak{g}} \omega$, is a twisted triangular structure on $\mathfrak{g}$. In this case, $\chi_{\mathfrak{p}, \mathfrak{g} / \mathfrak{p}}=-\left(e_{11}^{*}+e_{22}^{*}\right)$ and $\theta(\mathfrak{g}, r, \psi)=-\left(e_{13}+e_{23}\right)$. 


\subsection{Twisted quasi-Frobenius Lie algebras}

There is a twisted version of the quasi-Frobenius Lie algebras. A pair $(\omega, \psi)$ is called a twisted quasi-Frobenius structure on a Lie algebra $\mathfrak{p}$ if $\omega$ is a non-degenerate 2-cochain on $\mathfrak{p}$ and $\psi$ is a 3-cocycle on $\mathfrak{p}$ such that $\mathrm{d}_{\mathfrak{p}} \omega=-\psi$. Then [78],

Proposition 12. The pair $(\omega, \psi)$ is a twisted quasi-Frobenius structure on a Lie algebra, $\mathfrak{g}$, if and only if $\left(\omega^{-1}, \psi\right)$ is a twisted triangular structure on $\mathfrak{g}$.

In this correspondence, quasi-Frobenius structures correspond to triangular structures, so this proposition extends Proposition 9.

\section{Modular classes of Poisson-Nijenhuis structures}

The modular classes of Poisson-Nijenhuis manifolds were introduced in [25] and further studied in [75]. The extension of the definition and properties of the modular classes to the case of Poisson-Nijenhuis structures on Lie algebroids was carried out by Caseiro [17]. Applications to the classical integrable systems, the Toda lattice among them, are to be found in $[25,4,17]$.

\subsection{Nijenhuis structures}

The Nijenhuis torsion of a $(1,1)$-tensor, $N \in \Gamma\left(T M \otimes T^{*} M\right)$, on a manifold $M$, is the $(1,2)$ tensor $[N, N]_{\mathrm{Fr}-\mathrm{Nij}}$ defined by

$$
\frac{1}{2}[N, N]_{\mathrm{Fr}-\mathrm{Nij}}(X, Y)=[N X, N Y]-N[N X, Y]-N[X, N Y]+N^{2}[X, Y],
$$

for $X$ and $Y \in \Gamma(T M)$. A $(1,1)$-tensor is called a Nijenhuis tensor if its Nijenhuis torsion vanishes. It is well known that powers (with respect to composition) of Nijenhuis tensors, considered as endomorphisms of the tangent bundle, are Nijenhuis tensors. When $N$ is a $(1,1)$ tensor, set

$$
[X, Y]_{N}=[N X, Y]+[X, N Y]-N[X, Y]
$$

for $X$ and $Y \in \Gamma(T M)$.

Proposition 13. If $N$ is a Nijenhuis tensor on a manifold, $M$, the bracket $[,]_{N}$ defined by (8.1) is a Lie bracket on $\Gamma(T M)$, and the vector bundle $T M$ with anchor $N: T M \rightarrow T M$ and Lie bracket of sections $[,]_{N}$ is a Lie algebroid.

Let us denote the Lie algebroid $\left(T M, N,[,]_{N}\right)$ by $T M_{N}$. The Lie algebroid differential of $T M_{N}$ on $\Gamma\left(\wedge^{\bullet} T^{*} M\right)$ is $\mathrm{d}_{N}=\left[\mathrm{d}, \iota_{N}\right]$, where $\mathrm{d}$ is the de Rham differential and $\iota_{N}$ is the interior product of 1 -forms by the (1,1)-tensor $N$ [74]. The following proposition is from [25] (see also [75]).

Proposition 14. The modular class of the Lie algebroid $T M_{N}$ is the class of the 1-form $\mathrm{d} \operatorname{Tr} N$ in the $\mathrm{d}_{N}$-cohomology.

It follows either from the relation $[N, N]_{\mathrm{Fr}-\mathrm{Nij}}=0$, or from the fact that $N$ is the anchor of $T M_{N}$, that $N$ is a Lie algebroid morphism from $T M_{N}$ to $T M$. Since the modular class of $T M$ vanishes, the modular class of the Lie algebroid $T M_{N}$ is equal to the modular class of the Lie algebroid morphism $N$. 


\subsection{Poisson-Nijenhuis structures}

If $\pi$ is a Poisson bivector on $M$, we denote the Lie algebroid $\left(T^{*} M, \pi^{\sharp},[,]_{\pi}\right)$ by $T^{*} M_{[0]}$. A Nijenhuis tensor $N$ and a Poisson tensor $\pi$ on $M$ are called compatible if $\left(T M_{N}, T^{*} M_{[0]}\right)$ is a Lie bialgebroid. The pair $(\pi, N)$ is then called a Poisson-Nijenhuis structure (or PN-structure for short) on $M$, and $M$ is called a Poisson-Nijenhuis manifold.

This characterization of Poisson-Nijenhuis structures [65] is equivalent to the original definition [96, 74]: $\pi^{\sharp} N^{*}=N \pi^{\sharp}$ and $C(\pi, N)=0$, where $N^{*}: T^{*} M \rightarrow T^{*} M$ is the dual of $N: T M \rightarrow T M$, and $C(\pi, N)$ is a $(2,1)$-tensor defined by $C(\pi, N)(\alpha, \beta)=[\alpha, \beta]_{N \pi}-\left([\alpha, \beta]_{\pi}\right)_{N^{*}}$, for $\alpha$ and $\beta \in \Gamma\left(T^{*} M\right)$.

The main consequence of the compatibility of $\pi$ and $N$ is that all the bivectors in the sequence $\pi_{k}=N^{k} \pi, k \in \mathbb{N}$, are Poisson bivectors. In addition, $\left(\pi_{k}\right)$ constitutes a hierarchy of pairwise compatible Poisson structures in the sense that, for any $k, k^{\prime} \in \mathbb{N}, \pi_{k}+\pi_{k^{\prime}}$ is a Poisson bivector.

Define

$$
I_{k}=\frac{1}{k} \operatorname{Tr} N^{k},
$$

for each $k \geq 1$. The functions $I_{k}$ are pairwise in involution with respect to any of the Poisson structures $\pi_{\ell}$ [96], and they are called the fundamental functions in involution of the PoissonNijenhuis structure [96]. The corresponding Hamiltonian vector fields,

$$
H_{I_{k}}^{\pi}=\frac{1}{k} \pi^{\sharp}\left(\mathrm{d} \operatorname{Tr} N^{k}\right),
$$

are bi-Hamiltonian for $k \geq 2$ with respect to any pair of Poisson structures in the hierarchy, and they commute pairwise. The sequence of vector fields $\left(H_{I_{k}}^{\pi}\right)$ is called the bi-Hamiltonian hierarchy of the Poisson-Nijenhuis structure.

When $(\pi, N)$ is a Poisson-Nijenhuis structure on $M$, the vector bundle $T^{*} M$ with anchor $\left(\pi_{k}\right)^{\sharp}$ and bracket $[,]_{\pi_{k}}$ is a Lie algebroid which we denote by $T^{*} M_{[k]}$. We then denote $N^{*}$, considered as a map from $T^{*} M_{[k]}$ to $T^{*} M_{[k-1]}$, by $N_{[k]}^{*}$, for each $k \geq 1$.

Proposition 15. Let $(\pi, N)$ be a Poisson-Nijenhuis structure on $M$. Then, for all $k \geq 1$, $N_{[k]}^{*}: T^{*} M_{[k]} \rightarrow T^{*} M_{[k-1]}$ is a Lie algebroid morphism.

\subsection{The modular classes of a Poisson-Nijenhuis manifold}

Definition 7. For $k \geq 1$, the $k$-th modular class of a Poisson-Nijenhuis manifold $(M, \pi, N)$ is one-half of the modular class of the Lie algebroid morphism $N_{[k]}^{*}$.

We denote the $k$-th modular class of $(M, \pi, N)$ by $\theta^{(k)}$. We recall that, corresponding to each Poisson structure $\pi_{k}$ on $M$ and consequently to each Lie algebroid $T^{*} M_{[k]}$, there is associated a differential on $\Gamma\left(\wedge^{\bullet} T M\right)$, denoted by $\mathrm{d}_{\pi_{k}}$. The $k$-th modular class is a class in the first cohomology space of the complex $\left(\Gamma\left(\wedge^{\bullet} T M\right), \mathrm{d}_{\pi_{k}}\right)$.

Assume that $M$ is orientable, and let $\lambda$ be a volume form on $M$. For each $k \in \mathbb{N}$, let $X_{\lambda}^{k}$ be the modular vector field of the Poisson manifold $\left(M, \pi_{k}\right)$ which is a $\mathrm{d}_{\pi_{k}}$-cocycle. Then [25],

Theorem 6. Let $(M, \pi, N)$ be an orientable Poisson-Nijenhuis manifold. Set

$$
X^{(k)}=X_{\lambda}^{k}-N\left(X_{\lambda}^{k-1}\right),
$$

for each $k \geq 1$. Then,

- $X^{(k)}$ is a $\mathrm{d}_{\pi_{k}}$-cocycle, independent of the choice of the volume form, $\lambda$,

- the class of $X^{(k)}$ in the $\mathrm{d}_{\pi_{k}}$-cohomology of $\Gamma\left(\wedge^{\bullet} T M\right)$ is $\theta^{(k)}$, the $k$-th modular class of $(M, \pi, N)$,

- $X^{(k+1)}=N\left(X^{(k)}\right)$,

- $X^{(k)}=-\frac{1}{2} H_{I_{k}}^{\pi}$. 
The vector fields $X^{(k)}$ can therefore be called the modular vector fields of the PoissonNijenhuis structure $(\pi, N)$ on $M$. The sequence of modular vector fields $\left(X^{(k)}\right)_{k \geq 2}$ coincides, up to sign and a factor 2, with the bi-Hamiltonian hierarchy of the Poisson-Nijenhuis structure. Under the additional assumption that $N$ is invertible, each $X^{(k)}$ is bi-Hamiltonian, not only for $k \geq 2$, but also for $k=1$ with $I_{0}=\ln |\operatorname{det} N|$ (see [25]).

Remark 7. A proof of Theorem 6 is also in [75], based on the fact that $C(\pi, N)=0$ implies $\iota_{\pi}\left(\mathrm{d}_{N} \mathrm{~d} f\right)=-\frac{1}{2} H_{I_{1}}^{\pi}(f)$ which fact is a corollary of a theorem of Beltrán and Monterde [8].

The vector field $X^{(1)}=X_{\lambda}^{N \pi}-N\left(X_{\lambda}^{\pi}\right)$ is called the modular vector field of $(M, \pi, N)$. Its class in the $\mathrm{d}_{N \pi^{-}}$-cohomology is $\theta^{(1)}=\frac{1}{2} \operatorname{Mod}\left(N^{*}\right)$, called the modular class of $(M, \pi, N)$. Each $X^{(k)}$, for $k \geq 2$, is obtained from $X^{(1)}$ by the relation,

$$
X^{(k)}=N^{k-1} X^{(1)} .
$$

Let $T M_{[k]}$ be the Lie algebroid $\left(T M, N^{k},[,]_{N^{k}}\right)$, and let $N_{[k]}$ be $N$ considered as a Lie algebroid morphism from $T M_{[k]}$ to $T M_{[k-1]}$. There is a simple relation between the modular classes of the Lie algebroid morphisms $N_{[k]}^{*}: T^{*} M_{[k]} \rightarrow T^{*} M_{[k-1]}$ and $N_{[k]}: T M_{[k]} \rightarrow T M_{[k-1]}$.

Proposition 16. When $(\pi, N)$ is a Poisson-Nijenhuis structure on $M$,

$$
\operatorname{Mod}\left(N_{[k]}^{*}\right)=-\left(\pi^{\sharp}\right)^{*}\left(\operatorname{Mod}\left(N_{[k]}\right)\right),
$$

for all $k \geq 1$.

\subsection{Poisson-Nijenhuis structures on Lie algebroids}

It is straightforward to extend the notion of Poisson-Nijenhuis manifold to that of PoissonNijenhuis structure on a Lie algebroid [74, 47], more generally on Gelfand-Dorfman complexes $[40,27,119,120]$, and the results concerning the modular classes are readily extended [17]. For a Nijenhuis tensor on a Lie algebroid $A$, the modular class of the morphism $N$ from $A$ with anchor $a_{A} \circ N$ and deformed bracket $[,]_{A, N}$ to $\left(A, a_{A},[,]_{A}\right)$ is the class of the 1 -form $\mathrm{d}_{A} \operatorname{Tr} N$ (cf. Proposition 14). For a Poisson-Nijenhuis structure on $A$, there is a sequence of bi-Hamiltonian modular sections (cf. Theorem 6), and a multi-Hamiltonian system on the base manifold. In [17], Caseiro defines a Poisson-Nijenhuis structure on a Lie algebroid, whose hierarchy of Poisson structures projects onto the known hierarchy of multi-Hamiltonian structures for the Toda lattice.

\section{The spinor approach to the modular class}

The spinor approach to Poisson geometry stems from Hitchin's introduction of the generalized geometry using the Courant bracket on the direct sum $T M \oplus T^{*} M$ [51]. It was developed by Gualtieri [48], and, more recently by Alekseev, Bursztyn and Meinrenken [97, 5], and many others. Here we show how the modular class appears in this approach.

\subsection{The modular class and pure spinors}

The title of this section will be explained in the next section.

We first make two definitions. If $\pi$ is a bivector on a vector bundle, $E$, we denote by $e^{-\iota_{\pi}}$ the operator Id $-\iota_{\pi}+\frac{1}{2 !} \iota_{\pi} \circ \iota_{\pi}-\cdots+(-1)^{k} \frac{1}{k !}\left(\iota_{\pi}\right)^{k}+\cdots$, whose evaluation on a given form is a finite sum, and, for $\lambda$ a form of top degree on $E$, let us set

$$
\lambda^{(\pi)}=e^{-\iota \pi} \lambda .
$$


Next, we define, for $\psi$ a $\mathrm{d}_{A}$-closed 3 -form on a Lie algebroid $A$, the operator on forms,

$$
\mathrm{d}_{\psi}=\mathrm{d}_{A}+\epsilon_{\psi},
$$

where $\epsilon_{\psi}$ denotes the exterior product by $\psi$. This operator is of square zero since $\psi$ is $\mathrm{d}_{A}$-closed. Such a closed 3 -form is usually considered as a background 3 -form $[62,113,48]$, and $\mathrm{d}_{\psi}$ is called the $\psi$-twisted differential associated to $(A, \psi)$. We now show the relationship of these objects with the modular class.

Let $\pi$ be a bivector, $\psi$ a $\mathrm{d}_{A}$-closed 3-form, and $\lambda$ a volume form on an orientable Lie algebroid, $A$, and let $X_{\lambda}$ and $Y$ be defined as in Section $7.2, *_{\lambda} X_{\lambda}=-\partial_{\pi} \lambda$ and $Y=\pi^{\sharp} \iota_{\pi} \psi$. Then,

$$
\mathrm{d}_{A}\left(\lambda^{(\pi)}\right)=\iota_{-\frac{1}{2}[\pi, \pi]_{A}+X_{\lambda}}\left(\lambda^{(\pi)}\right),
$$

and

$$
\epsilon_{\psi}\left(\lambda^{(\pi)}\right)=\iota_{\left(\wedge^{3} \pi^{\sharp}\right) \psi+Y}\left(\lambda^{(\pi)}\right) .
$$

Setting $\phi_{\pi}=\frac{1}{2}[\pi, \pi]_{A}-\left(\wedge^{3} \pi^{\sharp}\right) \psi$ and $Z_{\lambda}=X_{\lambda}+Y$, we obtain

$$
\mathrm{d}_{\psi}\left(\lambda^{(\pi)}\right)=\iota_{-\phi_{\pi}+Z_{\lambda}}\left(\lambda^{(\pi)}\right) .
$$

These formulas are results of Meinrenken [97], themselves based on formulas of Evens and Lu [33]. (See also [7].) Since $\phi_{\pi}=0$ is the condition for $(\pi, \psi)$ to define a twisted Poisson structure on $A$, the following theorem is a direct consequence of (9.2).

Theorem 7. Let $(A, \pi, \psi)$ be an orientable Lie algebroid with a twisted Poisson structure, and let $\lambda$ be a volume form on $A$. The modular section associated to $\lambda$ is the unique section $Z_{\lambda}$ of $A$ such that

$$
\mathrm{d}_{\psi}\left(\lambda^{(\pi)}\right)=\iota_{Z_{\lambda}}\left(\lambda^{(\pi)}\right) .
$$

An orientable Lie algebroid with a twisted Poisson structure, $(A, \pi, \psi)$, is unimodular if and only if there exists a volume form, $\lambda$, on $A$ such that $\lambda^{(\pi)}$ is a $\mathrm{d}_{\psi}$-closed form.

If, in particular, $\psi=0$, equation (9.2) reduces to (9.1), and the theorem states that, for $(A, \pi)$ an orientable Lie algebroid with a Poisson structure, $\mathrm{d}_{A}\left(\lambda^{(\pi)}\right)=\iota_{X_{\lambda}}\left(\lambda^{(\pi)}\right)$, so that the unimodularity corresponds to the existence of a volume form, $\lambda$, such that $\lambda^{(\pi)}$ is $\mathrm{d}_{A}$-closed.

Remark 8. In terms of the big bracket, if we again denote the Lie algebroid structure of $A$ by $\mu$, relation (9.3) becomes $\left\{\mu, \lambda^{(\pi)}\right\}+\epsilon_{\psi}\left(\lambda^{(\pi)}\right)=\left\{Z_{\lambda}, \lambda^{(\pi)}\right\}$, and in the Poisson case, it takes the simple form, $\left\{\mu, \lambda^{(\pi)}\right\}=\left\{X_{\lambda}, \lambda^{(\pi)}\right\}$. Thus, for a Lie algebroid with a twisted Poisson structure, the modular section associated to the volume form $\lambda$ is the unique section, $Z$, of $A$ satisfying the equation,

$$
\left\{\mu, \lambda^{(\pi)}\right\}+\epsilon_{\psi}\left(\lambda^{(\pi)}\right)=\left\{Z, \lambda^{(\pi)}\right\},
$$

and, for a Poisson structure, this equation takes the simple form, $\left\{\mu, \lambda^{(\pi)}\right\}=\left\{X, \lambda^{(\pi)}\right\}$.

\subsection{Pure spinors}

When the sections of $\wedge^{\bullet}\left(E \oplus E^{*}\right)$ act on the sections of $\wedge^{\bullet}\left(E^{*}\right)$ by interior and exterior products, $\Gamma\left(\wedge^{\bullet} E^{*}\right)$ appears as a spinor bundle of the Clifford algebra of $E \oplus E^{*}$, because of the relation $\iota_{X} \epsilon_{\xi}+\epsilon_{\xi} \iota_{X}=\langle\xi, X\rangle$, for $X \in \Gamma E$ and $\xi \in \Gamma\left(E^{*}\right)$. If $\pi$ is a bivector on $E$, its graph is a Lagrangian sub-bundle of $E \oplus E^{*}$, i.e., a maximal isotropic sub-bundle with respect to the bilinear form defined by the pairing of $E$ with $E^{*}$. A Lagrangian sub-bundle $L$ of $E \oplus E^{*}$ is determined by a form, $\kappa \in \Gamma\left(\wedge^{\bullet} E^{*}\right)$, unique up to a multiplicative constant, such that $L$ is the annihilator of $\kappa$ under the Clifford action [21]. Such a form is called a pure spinor. It can de deduced from a theorem of Chevalley [21] that the graph of $\pi$ is the annihilator of the pure spinor $\lambda^{(\pi)}$. Thus, in the spinor approach to Poisson and twisted Poisson structures, the inhomogeneous form $\lambda^{(\pi)}$ is viewed as a pure spinor defining the graph of $\pi$. 


\subsection{Courant algebroids and Dirac structures}

When $A$ is a Lie algebroid, the vector bundle $A \oplus A^{*}$ becomes a Courant algebroid with the non-skewsymmetric bracket defined as a derived bracket of the big bracket by

$$
[X+\xi, Y+\eta]=\left\{\left\{X+\xi, \mathrm{d}_{A}\right\}, Y+\eta\right\},
$$

for $X, Y \in \Gamma A$ and $\xi, \eta \in \Gamma\left(A^{*}\right)$, and, more generally, if $\psi$ is a $d_{A^{-c l o s e d}} 3$-form on $A$, the vector bundle $A \oplus A^{*}$ is a Courant algebroid with the non-skewsymmetric bracket,

$$
[X+\xi, Y+\eta]_{\psi}=\left\{\left\{X+\xi, \mathrm{d}_{\psi}\right\}, Y+\eta\right\},
$$

called the Courant bracket with background $\psi$, or the $\psi$-twisted Courant bracket (see $[111,69,70]$ and, for further developments, [115, 71]).

A Lagrangian sub-bundle of a Courant algebroid whose sections are closed under the Courant bracket is called a Dirac structure.

By (9.2), requiring that $\phi_{\pi}=0$, the condition for $(\pi, \psi)$ to be a twisted Poisson structure, is equivalent to the condition that there exist a section $U$ of $A$ such that the pure spinor $\lambda^{(\pi)}$ satisfy

$$
\mathrm{d}_{\psi}\left(\lambda^{(\pi)}\right)=\iota_{U} \lambda^{(\pi)} .
$$

This condition, in turn, is equivalent to the condition that the annihilator of $\lambda^{(\pi)}$, a Lagrangian sub-bundle of $A \oplus A^{*}$, be closed under the Courant bracket with background $\psi$ [48, 97]. Since the annihilator of $\lambda^{(\pi)}$ is the graph of $\pi$, these considerations imply the following proposition.

Proposition 17. The pair $(\pi, \psi)$ is a twisted Poisson structure on $A$ if and only if the graph of $\pi$ is a Dirac sub-bundle of $A \oplus A^{*}$ with the $\psi$-twisted Courant bracket.

This proposition is well-known and can be proved without any appeal to spinors (see [113, $111,115])$. Here, we emphasize that the modular field appears as the section $U$ such that the $\psi$-twisted differential of $\lambda^{(\pi)}$ satisfies (9.5), which ensures that the graph of $\pi$ is closed under the $\psi$-twisted Courant bracket (9.4).

\section{Appendix: additional references and conclusion}

In this Appendix, we review some recent publications and the direction of work in progress.

Many lines of research that touch upon the subject of this survey have been or are being developed. In the early 90's, the modular vector fields were a tool in the classification of Poisson structures, in particular of the quadratic Poisson structures on the plane [31, 89, 46]. Work on the classification of Poisson structures on surfaces using the periods of a modular vector field along the singular set of the Poisson bivector has been carried out by Radko [109, 110]. Starting in 2000, results have been published on the relation of the modular class of a Poisson manifold and the holonomy of its symplectic foliation [44], the Godbillon-Vey class of the symplectic foliation in the regular case [99] and its Reeb class [1], and on the invariance of the modular class under Morita equivalence [43, 14]. In [59], Ibort and Martínez Torres study the unimodularity of some Poisson manifolds constructed by surgery. In [100], Mitric and Vaisman compute the modular class of the complete lift of the Poisson bivector of a manifold to its tangent bundle. In [126], Ping $\mathrm{Xu}$ determines how the modular class of a Dirac submanifold of a Poisson manifold is related to that of the ambient Poisson manifold. J.-H. Lu recently described the modular classes of Poisson homogeneous spaces [91], generalizing a result of [34].

At the same time many generalizations of the notion of modular class have appeared. The modular classes of Leibniz algebroids [58, 123], of Nambu-Poisson structures [57, 56], of Jacobi 
manifolds [121] and Jacobi algebroids (also called generalized Lie algebroids) [60] have been defined, their unimodularity implying a homology/cohomology duality, as well as those of symplectic supermanifolds [102]. The modular class of quasi-Poisson $G$-manifolds was defined by Alekseev et al. in [6].

Many further developments are in progress. To name just a few, work on the various generalizations continues, for example on Jacobi-Nijenhuis algebroids [18], while the implications of the properties of the modular classes of Poisson-Nijenhuis structures on manifolds and on Lie algebroids for the theory of integrable systems are being studied [4]. Closely related works are those of Launois and Richard on Poisson algebras [83], that of Dolgushev [26] on the exponentiation of the modular class into an automorphism of an associative, non-commutative algebra, quantizing a Poisson algebra, and that of Neumaier and Waldmann on the relationship between unimodularity and the existence of a trace in a quantized algebra [103]. The relationship between unimodularity and the existence of a trace on non-commutative algebras was already stressed by Weinstein in [124] and should be the subject of further work.

There are at least three directions in which the theory will surely expand: the definition and study of the modular classes of Lie groupoids and their behaviour under Morita equivalence, more generally introducing stacks and 2-categories (following, e.g., [15, 85, 116]), applications to sigma-models, and the determination of the relationship with generalized complex structures. The number of papers on sigma-models in which Lie algebroids and twisted Poisson structures appear is extremely large. In [11], Bonechi and Zabzine introduce the modular class of the target manifold of the Poisson sigma-model on the sphere: the unimodularity appears as a condition for the quantization to be well-defined. There is new work [84] on holomorphic Lie algebroids, and problems such as the relation between unimodularity and the generalized Calabi-Yau manifolds [51] remain to be studied.

\section{Acknowledgements}

I express my sincere gratitude to the organizers of the Midwest Geometry Conference in memory of Thomas Branson which was held at the University of Iowa in May 2007. Thomas Branson and I collaborated on an article in the early 80's, and the conference was a much appreciated opportunity to honor his memory and to learn about the considerable development of the topic in which we were both interested at the time, conformal invariance.

The present article incorporates many results due to Camille Laurent-Gengoux, Franco Magri, Alan Weinstein and Milen Yakimov, which have appeared in our joint publications and preprints. I would like to extend my sincere thanks to them for all they taught me, and I thank Camille, in particular, for his remarks on an early version of this paper. My thanks also to Tudor Ratiu for reminding me of the early history of the bracket of differential forms.

This survey takes into account most of the suggestions of the referees to whom I am most grateful for their very substantial and constructive comments.

\section{References}

[1] Abouqateb A., Boucetta M., The modular class of a regular Poisson manifold and the Reeb class of its symplectic foliation, C. R. Math. Acad. Sci. Paris 337 (2003), 61-66, math.DG/0211405.

[2] Abraham R., Marsden J.E., Foundations of mechanics, W.A. Benjamin, Inc., New York - Amsterdam, 1967.

[3] Abraham R., Marsden J.E., Foundations of mechanics, second edition, revised and enlarged, with the assistance of Tudor Raţiu and Richard Cushman, Benjamin/Cummings Publishing Co., Inc., Reading, Mass., 1978.

[4] Agrotis M., Damianou P., The modular hierarchy of the Toda lattice, Differential Geom. Appl., to appear, math.DG/0701057.

[5] Alekseev A., Bursztyn H., Meinrenken E., Pure spinors on Lie groups, arXiv:0709.1452. 
[6] Alekseev A., Kosmann-Schwarzbach Y., Meinrenken E., Quasi-Poisson manifolds, Canad. J. Math. 54 (2002), 3-29, math.DG/0006168.

[7] Alekseev A., Xu P., Derived brackets and Courant algebroids, unfinished manuscript, 2000.

[8] Beltrán J.V., Monterde J., Poisson-Nijenhuis structures and the Vinogradov bracket, Ann. Global Anal. Geom. 12 (1994), 65-78.

[9] Bhaskara K.H., Viswanath K., Calculus on Poisson manifolds, Bull. London Math. Soc. 20 (1988), 68-72.

[10] Bojowald M., Kotov A., Strobl T., Lie algebroid morphisms, Poisson sigma models, and off-shell closed gauge symmetries, J. Geom. Phys. 54 (2005), 400-426, math.DG/0406445.

[11] Bonechi F., Zabzine M., Poisson sigma model on the sphere, arXiv:0706.3164.

[12] Brylinski J.-L., A differential complex for Poisson manifolds, J. Differential Geom. 28 (1988), 93-114.

[13] Brylinski J.-L., Zuckerman G., The outer derivation of a complex Poisson manifold, J. Reine Angew. Math. 506 (1999), 181-189, math.DG/9802014.

[14] Bursztyn H., Radko O., Gauge equivalence of Dirac structures and symplectic groupoids, Ann. Inst. Fourier (Grenoble) $\mathbf{5 3}$ (2003), 309-337, math.SG/0202099.

[15] Bursztyn H., Weinstein A., Picard groups in Poisson geometry, Mosc. Math. J. 4 (2004), 39-66, math.SG/0304048.

[16] Cannas da Silva A., Weinstein A., Geometric models for noncommutative algebras, Berkeley Mathematics Lecture Notes, Vol. 10, Amer. Math. Soc., Providence, RI, 1999.

[17] Caseiro R., Modular classes of Poisson-Nijenhuis Lie algebroids, Lett. Math. Phys. 80 (2007), 223-238, math.DG/0701476.

[18] Caseiro R., Nunes da Costa J. M., Jacobi-Nijenhuis algebroids and their modular classes, arXiv:0706.1475.

[19] Chari V., Pressley A., A guide to quantum groups, Cambridge University Press, Cambridge, 1995.

[20] Chen Z., Liu Z.-J., On (co-)morphisms of Lie pseudoalgebras and groupoids, J. Algebra 316 (2007), 1-31, arXiv:0710.2149.

[21] Chevalley C., The algebraic theory of spinors, Columbia University Press, New York, 1954; The algebraic theory of spinors and Clifford algebras, Collected Works, Vol. 2, Springer-Verlag, Berlin, 1997.

[22] Cornalba L., Schiappa R., Nonassociative star product deformations for D-brane world-volumes in curved backgrounds, Comm. Math. Phys. 225 (2002), 33-66, hep-th/0101219.

[23] Coste A., Dazord P., Weinstein A., Groupoïdes symplectiques, Publications du Département de Mathématiques, Université Claude Bernard-Lyon I 2A (1987), 1-62.

[24] Crainic M., Differentiable and algebroid cohomology, van Est isomorphisms, and characteristic classes, Comment. Math. Helv. 78 (2003), 681-721, math.DG/0008064.

[25] Damianou P.A., Fernandes R.L., Integrable hierarchies and the modular class, math.DG/0607784.

[26] Dolgushev V., The Van den Bergh duality and the modular symmetry of a Poisson variety, math.QA/0612288.

[27] Dorfman I.Ya., Dirac structures and integrability of nonlinear evolution equations, John Wiley and Sons, Chichester, 1993.

[28] Douady A., Lazard M., Espaces fibrés en algèbres de Lie et en groupes, Invent. Math. 1 (1966), $133-151$.

[29] Drinfeld V.G., Hamiltonian structures on Lie groups, Lie bialgebras and the geometric meaning of classical Yang-Baxter equations, Dokl. Akad. Nauk SSSR 268 (1983), no. 2, 285-287 (English transl.: Sov. Math. Dokl. 27 (1983), no. 2, 68-71).

[30] Drinfeld V.G., Quasi-Hopf algebras, Algebra i Analiz 1 (1989), 114-148 (English transl.: Leningrad Math. J. 1 (1990), 1419-1457).

[31] Dufour J.-P., Haraki A., Rotationnels et structures de Poisson quadratiques, C. R. Acad. Sci. Paris Sér. I Math. 312 (1991), 137-140.

[32] Ehresmann C., Catégories topologiques et catégories différentiables, in Colloque Géom. Diff. Globale (1958, Bruxelles), Centre Belge Rech. Math., Louvain, 1959, 137-150.

[33] Evens S., Lu J.-H., Poisson harmonic forms, Kostant harmonic forms, and the $S^{1}$-equivariant cohomology of K/T, Adv. Math. 142 (1999), 171-220, dg-ga/9711019.

[34] Evens S., Lu J.-H., Weinstein A., Transverse measures, the modular class and a cohomology pairing for Lie algebroids, Quart. J. Math. Ser. 250 (1999), 417-436, dg-ga/9610008. 
[35] Fernandes R., Connections in Poisson geometry. I. Holonomy and invariants, J. Differential Geom. 54 (2000), 303-365, math.DG/0001129.

[36] Fernandes R., Lie algebroids, holonomy and characteristic classes, Adv. Math. 170 (2002), 119-179, math.DG/0007132.

[37] Fernandes R., Invariants of Lie algebroids, Differential Geom. Appl. 19 (2003), 223-243, math.DG/0202254.

[38] Flato M., Lichnerowicz A., Sternheimer D., Algèbres de Lie attachées a une variété canonique, J. Math. Pures Appl. (9) 54 (1975), 445-480.

[39] Fuchssteiner B., Lie algebra structure of degenerate Hamiltonian and bi-Hamiltonian systems, Progr. Theoret. Phys. 68 (1982), 1082-1104.

[40] Gelfand I.M., Dorfman I.Ya., Schouten bracket and Hamiltonian operators, Funktsional. Anal. $i$ Prilozhen. 14 (1980), no. 3, 71-74 (English transl.: Funct. Anal. Appl. 14 (1980), no. 3, 223-226).

[41] Gerstenhaber M., The cohomology structure of an associative ring, Ann. of Math. (2) 78 (1963), $267-288$.

[42] Gerstenhaber M., Giaquinto A., Boundary solutions of the classical Yang-Baxter equation, Lett. Math. Phys. 40 (1997), 337-353, q-alg/9609014.

[43] Ginzburg V.L., Grothendieck groups of Poisson vector bundles, J. Symplectic Geom. 1 (2001), 121-169, math.DG/0009124.

[44] Ginzburg V.L., Golubev A., Holonomy on Poisson manifolds and the modular class, Israel J. Math. 122 (2001), 221-242, math.DG/9812153.

[45] Grabowski J., Marmo G., Michor P., Homology and modular classes of Lie algebroids, Ann. Inst. Fourier (Grenoble) $\mathbf{5 6}$ (2006), 69-83, math.DG/0310072.

[46] Grabowski J., Marmo G., Perelomov A. M., Poisson structures: towards a classification, Modern Phys. Lett. A 8 (1993), 1719-1733.

[47] Grabowski J., Urbański P., Lie algebroids and Poisson-Nijenhuis structures, Rep. Math. Phys. 40 (1997), 195-208, dg-ga/9710007.

[48] Gualtieri M., Generalized complex geometry, math.DG/0703298.

[49] Higgins P.J., Mackenzie K., Algebraic constructions in the category of Lie algebroids, J. Algebra 129 (1990), $194-230$.

[50] Hirota Y., Morita invariant properties of twisted Poisson manifolds, Lett. Math. Phys. 81 (2007), $185-195$.

[51] Hitchin N., Generalized Calabi-Yau manifolds, Q. J. Math. 54 (2003), 281-308, math.DG/0209099.

[52] Hodges T., Yakimov M., Triangular Poisson structures on Lie groups and symplectic reduction, in Noncommutative Geometry and Representation Theory in Mathematical Physics, Editors J. Fuchs et al., Contemp. Math. 391 (2005), 123-134, math.SG/0412082.

[53] Huebschmann J., Poisson cohomology and quantization, J. Reine Angew. Math. 408 (1990), 57-113.

[54] Huebschmann J., Lie-Rinehart algebras, Gerstenhaber algebras, and Batalin-Vilkovisky algebras, Ann. Inst. Fourier (Grenoble) 48 (1998), 425-440, dg-ga/9704005.

[55] Huebschmann J., Duality for Lie-Rinehart algebras and the modular class, J. Reine Angew. Math. 510 (1999), 103-159, dg-ga/9702008.

[56] Ibáñez R., de León M., López B., Marrero J.C., Padrón E., Duality and modular class of a Nambu-Poisson structure, J. Phys. A: Math. Gen. 34 (2001), 3623-3650, math.SG/0004065.

[57] Ibáñez R., de León M., Marrero J.C., Padrón E., Leibniz algebroid associated with a Nambu-Poisson structure, J. Phys. A: Math. Gen. 32 (1999), 8129-8144, math-ph/9906027.

[58] Ibáñez R., Lopez B., Marrero J.C., Padrón E., Matched pairs of Leibniz algebroids, Nambu-Jacobi structures and modular class, C. R. Acad. Sci. Paris Sér. I Math. 333 (2001), 861-866.

[59] Ibort A., Martínez Torres D., A new construction of Poisson manifolds, J. Symplectic Geom. 2 (2003), 83-107.

[60] Iglesias D., Lopez B., Marrero J.C., Padrón E., Triangular generalized Lie bialgebroids: homology and cohomology theories, in Lie Algebroids and Related Topics in differential Geometry (2000, Warsaw), Banach Center Publ., Vol. 54, Polish Acad. Sci., Warsaw, 2001, 111-133.

[61] Karasëv M.V., Analogues of objects of the theory of Lie groups for nonlinear Poisson brackets, Izv. Akad. Nauk SSSR Ser. Mat. 50 (1986), no. 3, 508-538 (English transl.: Math. USSR Izv. 28 (1987), 497-527).

[62] Klimčík C., Strobl T., WZW-Poisson manifolds, J. Geom. Phys. 43 (2002), 341-344, math.SG/0104189. 
[63] Kosmann-Schwarzbach Y., Jacobian quasi-bialgebras and quasi-Poisson Lie groups, in Mathematical Aspects of Classical Field Theory (1991, Seattle, WA), Editors M.J. Gotay, J.E. Marsden and V. Moncrief, Contemp. Math. 132 (1992), 459-489.

[64] Kosmann-Schwarzbach Y., Exact Gerstenhaber algebras and Lie bialgebroids, Acta Appl. Math. 41 (1995), $153-165$.

[65] Kosmann-Schwarzbach Y., The Lie bialgebroid of a Poisson-Nijenhuis manifold, Lett. Math. Phys. 38 (1996), 421-428.

[66] Kosmann-Schwarzbach Y., From Poisson algebras to Gerstenhaber algebras, Ann. Inst. Fourier (Grenoble) 46 (1996), 1243-1274.

[67] Kosmann-Schwarzbach Y., Lie bialgebras, Poisson Lie groups and dressing transformations, in Integrability of nonlinear systems (1996, Pondicherry), Lecture Notes in Phys., Vol. 495, Springer, Berlin, 1997, $104-170$.

[68] Kosmann-Schwarzbach Y., Modular vector fields and Batalin-Vilkovisky algebras, in Poisson Geometry (1998, Warsaw), Editors J. Grabowski and P. Urbański, Banach Center Publ., Vol. 51, Polish Acad. Sci., Warsaw, 2000, 109-129.

[69] Kosmann-Schwarzbach Y., Derived brackets, Lett. Math. Phys. 69 (2004), 61-87, math.DG/0312524.

[70] Kosmann-Schwarzbach Y., Quasi, twisted, and all that ... in Poisson geometry and Lie algebroid theory, in The Breadth of Symplectic and Poisson Geometry, Editors J.E. Marsden and T. Ratiu, Progr. Math., Vol. 232, Birkhäuser, Boston, MA, 2005, 363-389, math.SG/0310359.

[71] Kosmann-Schwarzbach Y., Poisson and symplectic functions in Lie algebroid theory, arXiv:0711.2043.

[72] Kosmann-Schwarzbach Y., Laurent-Gengoux C., The modular class of a twisted Poisson structure, Trav. Math. 16 (2005), 315-339, math.SG/0505663.

[73] Kosmann-Schwarzbach Y., Laurent-Gengoux C., Weinstein A., Modular classes of Lie algebroid morphisms, arXiv:0712.3021.

[74] Kosmann-Schwarzbach Y., Magri F., Poisson-Nijenhuis structures, Ann. Inst. H. Poincaré Phys. Théor. 53 (1990), 35-81.

[75] Kosmann-Schwarzbach Y., Magri F., On the modular classes of Poisson-Nijenhuis manifolds, math.SG/0611202.

[76] Kosmann-Schwarzbach Y., Monterde J., Divergence operators and odd Poisson brackets, Ann. Inst. Fourier (Grenoble) $\mathbf{5 2}$ (2002), 419-456, math.QA/0002209.

[77] Kosmann-Schwarzbach Y., Weinstein A., Relative modular classes of Lie algebroids, C. R. Math. Acad. Sci. Paris 341 (2005), 509-514, math.DG/0508515.

[78] Kosmann-Schwarzbach Y., Yakimov M., Modular classes of regular twisted Poisson structures on Lie algebroids, Lett. Math. Phys. 80 (2007), 183-197, math.SG/0701209.

[79] Kostant B., Sternberg S., Symplectic reduction, BRS cohomology, and infinite-dimensional Clifford algebras, Ann. Physics 176 (1987), 49-113.

[80] Koszul J.-L., Crochet de Schouten-Nijenhuis et cohomologie, in The Mathematical Heritage of Élie Cartan (1984, Lyon), Astérisque (1985), 257-271.

[81] Kotov A., Strobl T., Characteristic classes associated to $Q$-bundles, arXiv:0711.4106.

[82] Kubarski J., The Weil algebra and the secondary characteristic homomorphism of regular Lie algebroids, in Lie Algebroids and Related Topics in Differential Geometry (2000, Warsaw), Banach Center Publ., Vol. 54, Polish Acad. Sci., Warsaw, 2001, 135-173.

[83] Launois S., Richard L., Twisted Poincaré duality for some quadratic Poisson algebras, Lett. Math. Phys. 79 (2007), 161-174, math.KT/0609390.

[84] Laurent-Gengoux C., Stiénon M., Xu P., Holomorphic Poisson structures and groupoids, arXiv:0707.4253.

[85] Laurent-Gengoux C., Tu J.-L., Xu P., Chern-Weil map for principal bundles over groupoids, Math. Z. 255 (2007), 451-491, math.DG/0401420.

[86] Lecomte P., Roger C., Modules et cohomologies des bigèbres de Lie, C. R. Acad. Sci. Paris Sér. I Math. 310 (1990), 405-410, Erratum, 893-894.

[87] Libermann P., Marle C.-M., Symplectic geometry and analytical mechanics, Mathematics and Its Applications, Vol. 35, D. Reidel Publishing Co., Dordrecht, 1987.

[88] Lichnerowicz A., Les variétés de Poisson et leurs algèbres de Lie associées, J. Differential Geom. 12 (1977), 253-300. 
[89] Liu Z.J., Xu P., On quadratic Poisson structures, Lett. Math. Phys. 26 (1992), 33-42.

[90] Liu Z.J., Xu P., Exact Lie bialgebroids and Poisson groupoids, Geom. Funct. Anal. 6 (1996), 138-145.

[91] Lu J.-H., A note on Poisson homogeneous spaces, arXiv:0706.1337.

[92] Lyakhovich S.L., Sharapov A.A., Characteristic classes of gauge systems, Nuclear Phys. B 703 (2004), 419-453.

[93] Mackenzie K., Lie groupoids and Lie algebroids in differential geometry, London Mathematical Society Lecture Note Series, Vol. 124, Cambridge University Press, Cambridge, 1987.

[94] Mackenzie K., General theory of Lie groupoids and Lie algebroids, London Mathematical Society Lecture Note Series, Vol. 213, Cambridge University Press, Cambridge, 2005.

[95] Mackenzie K., Xu P., Lie bialgebroids and Poisson groupoids, Duke Math. J. 73 (1994), 415-452.

[96] Magri F., Morosi C., A geometrical characterization of integrable Hamiltonian systems through the theory of Poisson-Nijenhuis manifolds, Quaderno, Vol. 19, University of Milano, 1984.

[97] Meinrenken E., Lectures on pure spinors and moment maps, math.DG/0609319.

[98] Menichi L., Batalin-Vilkovisky algebra structures on Hochschild cohomology, arXiv:0711.1946.

[99] Mikami K., Godbillon-Vey classes of symplectic foliations, Pacific J. Math. 194 (2000), 165-174.

[100] Mitric G., Vaisman I., Poisson structures on tangent bundles, Differential Geom. Appl. 18 (2003), 207-228, math.DG/0108130.

[101] Moerdijk I., Mrčun J., Introduction to foliations and Lie groupoids, Cambridge Studies in Advanced Mathematics, Vol. 91, Cambridge University Press, Cambridge, 2003.

[102] Monterde J., Vallejo J. A., A modular class of even symplectic manifolds, Teoret. Mat. Fiz. 132 (2002), 50-59 (English transl.: Theoret. and Math. Phys. 132 (2002), 934-941).

[103] Neumaier N., Waldmann S., Deformation quantization of Poisson structures associated to Lie algebroids, arXiv:0708.0516.

[104] Palais R., The cohomology of Lie rings, in Proc. Sympos. Pure Math., Vol. 3, Amer. Math. Soc., Providence, RI, 1961, 130-137.

[105] Park J.-S., Topological open p-branes, in Symplectic Geometry and Mirror Symmetry (2000, Seoul), World Sci. Publ., River Edge, NJ, 2001, 311-384.

[106] Pestun V., Topological strings in generalized complex space, Adv. Theor. Math. Phys. 11 (2007), 399-450, hep-th/0603145.

[107] Polishchuk A., Algebraic geometry of Poisson brackets, in Algebraic Geometry 7, J. Math. Sci. (New York) 84 (1997), 1413-1444.

[108] Pradines J., Théorie de Lie pour les groupoïdes différentiables. Calcul différentiel dans la catégorie des groupoïdes infinitésimaux, C. R. Acad. Sci. Paris Sér. A-B 264 (1967), A245-A248.

[109] Radko O., A classification of topologically stable Poisson structures on a compact oriented surface, J. Symplectic Geom. 1 (2002), 523-542, math.SG/0110304.

[110] Radko O., Toward a classification of Poisson structures on surfaces, in Quantization, Poisson Brackets and Beyond (2001, Manchester), Editor T. Voronov, Contemp. Math. 315 (2002), 81-88.

[111] Roytenberg D., Quasi-Lie bialgebroids and twisted Poisson manifolds, Lett. Math. Phys. 61 (2002), 123-137, math.QA/0112152.

[112] Schwarz A., Geometry of Batalin-Vilkovisky quantization, Comm. Math. Phys. 155 (1993), 249-260, hep-th/9205088.

[113] Ševera P., Weinstein A., Poisson geometry with a 3-form background, in Noncommutative Geometry and String Theory, Editors Y. Maeda and S. Watamura, Progr. Theoret. Phys. Suppl. 144 (2001), 145-154.

[114] Stolin A., On rational solutions of Yang-Baxter equation for $\mathfrak{s l}(n)$, Math. Scand. 69 (1991), 57-80.

[115] Terashima Y., On Poisson functions, J. Symplectic Geom., to appear.

[116] Tseng H.-H., Zhu C., Integrating Lie algebroids via stacks, Compos. Math. 142 (2006), 251-270, math.DG/0405003.

[117] Vaintrob A.Yu., Lie algebroids and homological vector fields, Usp. Mat. Nauk 52 (1997), 161-162 (English transl.: Russ. Math. Surv. 52 (1997), 428-429).

[118] Vaisman I., Lectures on the geometry of Poisson manifolds, Progr. Math., Vol. 118, Birkhäuser, Basel, 1994. 
[119] Vaisman I., The Poisson-Nijenhuis manifolds revisited, Rend. Sem. Mat. Univ. Politec. Torino 52 (1994), 377-394.

[120] Vaisman I., A lecture on Poisson-Nijenhuis structures, in Integrable Systems and Foliations (1995, Montpellier), Editors C. Albert, R. Brouzet and J.-P. Dufour, Progr. Math., Vol. 145, Birkhäuser, Boston, MA, 1997, 169-185.

[121] Vaisman I., The BV-algebra of a Jacobi manifold, Ann. Polon. Math. 73 (2000), 275-290, math.DG/9904112.

[122] Voronov T., Graded manifolds and Drinfeld doubles for Lie bialgebroids, in Quantization, Poisson Brackets and Beyond (2001, Manchester), Editor T. Voronov, Contemp. Math. 315 (2002), 131-168, math.DG/0105237.

[123] Wade A., On some properties of Leibniz algebroids, in Infinite Dimensional Lie Groups in Geometry and Representation Theory (2000, Washington, DC), World Sci. Publ., River Edge, NJ, 2002, 65-78.

[124] Weinstein A., The modular automorphism group of a Poisson manifold, J. Geom. Phys. 23 (1997), 379-394.

[125] Xu P., Gerstenhaber algebras and BV-algebras in Poisson geometry, Comm. Math. Phys. 200 (1999), 545560, dg-ga/9703001.

[126] Xu P., Dirac submanifolds and Poisson involutions, Ann. Sci. École Norm. Sup. (4) 36 (2003), 403-430, math.SG/0110326.

[127] Zabzine M., Lectures on generalized complex geometry and supersymmetry, Arch. Math. (Brno) 42 (2006), suppl., 119-146, hep-th/0605148. 